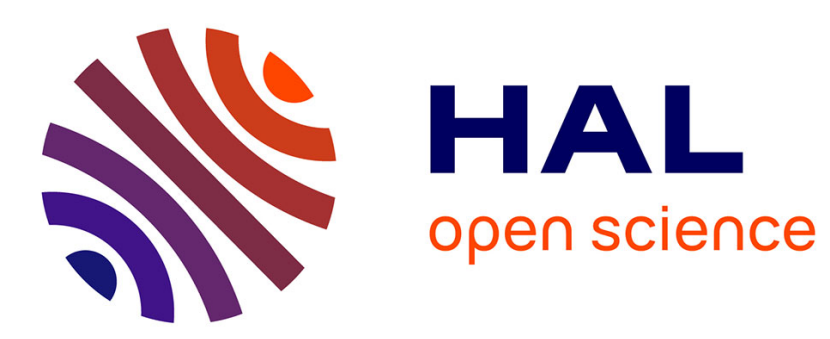

\title{
An analysis of composite beams by means of hierarchical finite elements and a variables separation method
}

\author{
O. Polit, L. Gallimard, P. Vidal, M. d'Ottavio, G. Giunta, S. Belouettar
}

\section{To cite this version:}

O. Polit, L. Gallimard, P. Vidal, M. d'Ottavio, G. Giunta, et al.. An analysis of composite beams by means of hierarchical finite elements and a variables separation method. Computers \& Structures, 2015, 158, pp.15 - 29. 10.1016/j.compstruc.2015.05.033 . hal-01366902

\section{HAL Id: hal-01366902 \\ https://hal.science/hal-01366902}

Submitted on 28 Oct 2019

HAL is a multi-disciplinary open access archive for the deposit and dissemination of scientific research documents, whether they are published or not. The documents may come from teaching and research institutions in France or abroad, or from public or private research centers.
L'archive ouverte pluridisciplinaire HAL, est destinée au dépôt et à la diffusion de documents scientifiques de niveau recherche, publiés ou non, émanant des établissements d'enseignement et de recherche français ou étrangers, des laboratoires publics ou privés. 


\title{
An analysis of composite beams by means of hierarchical finite elements and a variables separation method
}

\author{
O. Polit ${ }^{\text {a }}$, L. Gallimard ${ }^{\text {a }}$, P. Vidal ${ }^{\text {a }}$, M. D'Ottavio ${ }^{a}$, G. Giunta ${ }^{\text {b,*, S. Belouettar }}{ }^{\text {b }}$ \\ a Université Paris Ouest Nanterre-La Défense, 50 rue de Sèvres, 92410 Ville d'Avray, France \\ ${ }^{\mathrm{b}}$ Luxembourg Institute of Science and Technology, 5, avenue des Hauts-Fourneaux, L-4362 Esch-sur-Alzette, Luxembourg
}

Keywords:

Three-dimensional beam structures One-dimensional hierarchical modelling

Finite element modelling

Proper Generalised Decomposition

Composite materials

\begin{abstract}
A B S T R A C T
Laminated composite three-dimensional beams are investigated by means of hierarchical one-dimensional finite elements and a space decomposition approach. The global three-dimensional problem is separated by a Proper Generalised Decomposition into two coupled ones over the cross-section approximation space and on the beam axis approximation space. The size of the resulting problem is smaller than that of a classical equivalent finite element solution. The approximation of the displacements versus the cross-section and along the axis are general regardless the kinematic expansion order and the number of nodes per element thanks to a Unified Formulation. Accurate yet computationally affordable solutions are obtained.
\end{abstract}

\section{Introduction}

Over the last decades, composite materials have been extensively used in primary and secondary loading carrying structures due to high stiffness- and strength-to-weight ratios. Nevertheless, the resulting mechanical response is more complex than that of classical isotropic materials since a wider number of parameters (such as anisotropy as well as fibres and matrix geometry and material and stacking sequence) are involved. Furthermore, beam structures play an important role in many engineering fields such as aeronautics, space, automotive and civil construction and accurate models are required. Composite beam modelling, therefore, is an important and up-to-date research topic.

Classical models for the analysis of beams under bending are based upon Euler-Bernoulli's and Timoshenko's kinematic hypotheses: the cross-section is supposed to be rigid on its plane and the shear deformation is either constant over the cross-section (Timoshenko's model) or neglected (Euler-Bernoull i's theory). Furthermore, the material stiffness coefficients in Hooke's law should be opportunely corrected due to the Poisson locking, see Giunta et al. [1]. These models do not yield accurate results in several cases where composite laminated materials are used and, over the last years, many improvements in classical beam models have been proposed. In this sense, Kapania and Raciti [2,3] proposed a literature survey for the static,

\footnotetext{
* Corresponding author. Tel.: +352 545580 479; fax: +352 425991555 E-mail address: gaetano.giunta@list.lu (G. Giunta).
}

free-vibration, stability and wave propagation analyses of beams. Ghugal and Shimpi [4] addressed many equivalent single layer and layer-wise refined models and Chakravarty [5] discussed several ways for composite beam cross-section analysis. A finite element with a cubic variation of the displacement components over the thickness was presented by Maiti and Sinha [6] for the bending and free vibration analysis of laminated beams. Shimpi and Ghugal [7] formulated a layer-wise trigonometric shear deformation theory where a first-order shear deformation approximation is used for each layer. The theory was improved by reducing the number of primary variables, see [8]. A global higher-order theory was presented by Matsunaga [9] to investigate composite beams subjected to transverse loadings. Material constitutive relations were used to retrieve the axial stresses, whereas the transverse components were determined by integration of the three-dimensional equilibrium equations. Hodges et al. [10] and Yu et al. [11] proposed to model composite beams by means of a variational asymptotic beam section analysis where no cross-section a priori assumption is assumed. The general three-dimensional anisotropic elasticity problem is decoupled into a non-linear one-dimensional beam analysis and a linear two-dimensional cross-section analysis. A Timoshenko-like model is, then, obtained by reproducing the three-dimensional strain energy in a classical one-dimensional approximation space resulting in an enhanced model since in- and out-of-plane warping are accounted for. Vo and Lee [12] investigated the non-linear static response of thin-walled composite beams by a first-order shear deformable one-dimensional beam finite element. Geometrical 
non-linearities were accounted for in a Von Kármán sense. Lin and Zhang [13] developed a two-node beam element based upon Timoshenko's beam theory. Each node has two degrees of freedom: the transverse displacement and the cross-section rotation. The element, therefore, can be used for the investigation of axial-bending uncoupled laminates. Displacements' shape functions are derived from Timoshenko's composite beam functions. Classical Euler-Bernoulli's and Timoshenko's as well as secondand third-order beam models were used by Aguiar et al. [14] to derived finite elements based upon displacement, mixed and mixed least-squares formulations. Vo and Thai [15] formulated a two-node $\mathrm{C}^{1}$ element with six degrees of freedom per node. Classical theories, polynomial third-order and sinusoidal approximations were used for the axial displacement along the thickness direction. In the case of the higher-order models, top and bottom stress free boundary conditions are satisfied by a parabolic variation of the shear strains. Erkmen [16] proposed a shear deformable finite element where Timoshenko's theory was assumed locally at each layer and continuity was recovered by imposing multiple-point constraint together with a variational multi-scale approach. The element was, then, used to investigate composite beams with partially bonded layers.

Higher-order models usually come at the price of high computational costs. Within this paper, accurate yet efficient one-dimensional finite elements are derived for the static analysis of three-dimensional composite beams. The kinematic field is axiomatically assumed over the cross-section via a Unified Formulation (UF). This UF was previously derived for plates and shells (see Carrera [17] and Carrera and Giunta [18]) and, then, extended to the analysis of beam structures, see Carrera et al. [19], Giunta et al. [20] and Catapano et al. [21]. Several higher-order models can be straightforwardly obtained via this approach. The weak form of the governing differential equations is derived through the Principle of Virtual Displacement (PVD) in terms of a "fundamental nucleo" that does not depend upon the displacement field approximation order over the cross-section nor upon the number of nodes per element along the beam axis. Displacement-based theories accounting for non-classical effects, such as transverse shear and cross-section in- and out-of-plane warping, are derived.

Computational costs can become prohibitive when the number of elements and/or the approximation order increase often resulting in a stack overflow. An "out-of-core" computation approach (as used in commercial FEM packages and several linear algebra libraries) is a possible solution to this problem: part of the static (and slow) memory of the hard disk is used, see Castellanos and Larrazabal [22]. A model reduction approach where the approximation space is divided into coupled smaller ones is instead used in this paper, see Ammar et al. [23]. The variable separation technique is based on a Proper Generalised Decomposition (PGD). The need in memory is considerably reduced by decoupling the approximation over the beam cross-section from that along the axis and solving two coupled systems whose size is considerably smaller than that of the corresponding classical finite element solution. In this manner, the calculations are all performed "in-core". Nouy [24] and Chinesta et al. [25] presented a detailed review of the PGD and its application in different engineering fields. One of the first application of the PDG to structural mechanics, to the best of authors' knowledge, can be found in Savoia and Reddy [26] and, more recently, in Bognet et al. [27]. Vidal and co-workers investigated two-dimensional laminated and sandwich beams [28,29], laminated and sandwich plates [30,31] and cylindrical composite shells [32] within a PGD framework. Moreover, a reliability analysis in terms of failure probability of multi-layered two-dimensional structures was carried out by Gallimard et al. [33].
The derived one-dimensional finite elements are used to study laminated slender and short beams with prismatic or hollow cross-sections under bending or both bending and torsion. Results are compared to solutions obtained via a closed-form Navier-type method as well as corresponding one-dimensional finite elements in a classical sense. Three-dimensional finite element solutions using ANSYS are also presented. Numerical results show that accurate results can be obtained with a reduced computational effort when compared with the corresponding one-dimensional finite elements as well the three-dimensional solutions.

\section{Preliminaries}

A beam is a structure whose axial extension $(l)$ is predominant with respect to any other dimension orthogonal to it. The cross-section $(\Omega)$ is defined by intersecting the beam with planes orthogonal to its axis. A Cartesian reference system is adopted. The $x$ coordinate is coincident with the axis of the beam and it is bounded such that $0 \leqslant x \leqslant l$, whereas the $y$ - and $z$-axes are two orthogonal directions laying on $\Omega$. Fig. 1 presents the beam geometry and the reference system. The displacement field is:

$\mathbf{u}^{T}(x, y, z)=\left\{u_{x}(x, y, z) \quad u_{y}(x, y, z) \quad u_{z}(x, y, z)\right\}$

where $u_{x}, u_{y}$ and $u_{z}$ are the displacement components along the $x$-, $y$ - and $z$-axis, respectively. Superscript ' $T$ ' represents the transposition operator. Stresses $(\boldsymbol{\sigma})$ are grouped into vectors $\boldsymbol{\sigma}_{n} \in \mathbb{R}^{3}$ acting on the cross-section:

$\boldsymbol{\sigma}_{n}^{T}=\left\{\begin{array}{lll}\sigma_{x x} & \sigma_{x y} & \sigma_{x z}\end{array}\right\}$

and $\boldsymbol{\sigma}_{p} \in \mathbb{R}^{3}$ acting on planes orthogonal to $\Omega$ :

$\boldsymbol{\sigma}_{p}^{T}=\left\{\begin{array}{lll}\sigma_{y y} & \sigma_{z z} & \sigma_{y z}\end{array}\right\}$

The associated strains, $\boldsymbol{\varepsilon}_{n}$ and $\boldsymbol{\varepsilon}_{p}$, are:

$\boldsymbol{\varepsilon}_{n}^{T}=\left\{\begin{array}{lll}\varepsilon_{x x} & \gamma_{x y} & \gamma_{x z}\end{array}\right\} \quad \boldsymbol{\varepsilon}_{p}^{T}=\left\{\begin{array}{lll}\varepsilon_{y y} & \varepsilon_{z z} & \gamma_{y z}\end{array}\right\}$

In the case of small displacements, linear relations between strains and displacements hold:

$\boldsymbol{\varepsilon}_{n}^{T}=\left\{\begin{array}{lll}u_{x, x} & u_{x, y}+u_{y, x} & u_{x, z}+u_{z, x}\end{array}\right\}$

$\boldsymbol{\varepsilon}_{p}^{T}=\left\{\begin{array}{lll}u_{y, y} & u_{z, z} & u_{y, z}+u_{z, y}\end{array}\right\}$

Subscripts ' $x$ ', ' $y$ ' and ' $z$ ', when preceded by comma, represent derivation versus the corresponding spatial coordinate. Eq. (5) in a compact vectorial notation read:

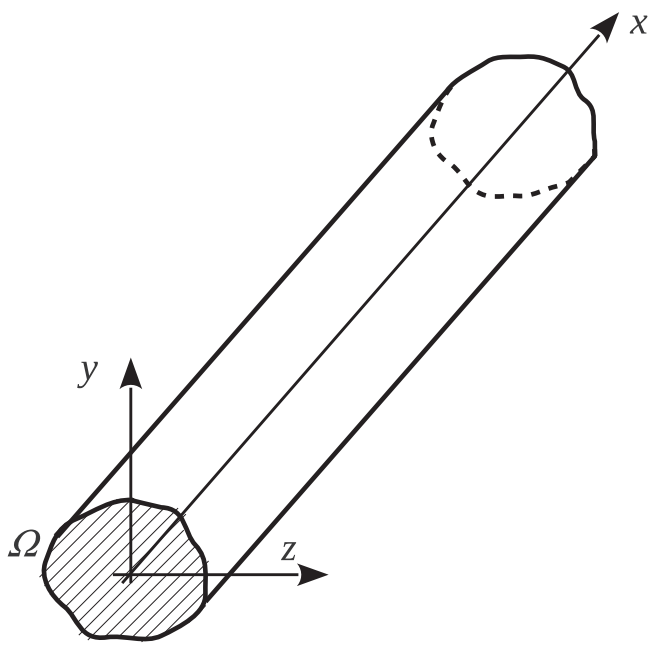

Fig. 1. Beam geometry and reference system. 
$\boldsymbol{\varepsilon}_{n}=\mathbf{D}_{n p} \mathbf{u}+\mathbf{D}_{n x} \mathbf{u}$

$\boldsymbol{\varepsilon}_{p}=\mathbf{D}_{p} \mathbf{u}$

$\mathbf{D}_{n p}, \mathbf{D}_{n x}$ and $\mathbf{D}_{p}$ are the following differential matrix operators:

$\mathbf{D}_{n p}=\left[\begin{array}{ccc}0 & 0 & 0 \\ \frac{\partial}{\partial y} & 0 & 0 \\ \frac{\partial}{\partial z} & 0 & 0\end{array}\right] \quad \mathbf{D}_{n x}=\mathbf{I} \frac{\partial}{\partial x} \quad \mathbf{D}_{p}=\left[\begin{array}{ccc}0 & \frac{\partial}{\partial y} & 0 \\ 0 & 0 & \frac{\partial}{\partial z} \\ 0 & \frac{\partial}{\partial z} & \frac{\partial}{\partial y}\end{array}\right]$

being $\mathbf{I} \in \mathbb{R}^{3 \times 3}$ the unit matrix.

Under the hypothesis of linear elastic materials, the constitutive relations are:

$\begin{aligned} \boldsymbol{\sigma}_{p} & =\tilde{\mathbf{C}}_{p p}^{k} \boldsymbol{\varepsilon}_{p}+\tilde{\mathbf{C}}_{p n}^{k} \boldsymbol{\varepsilon}_{n} \\ \boldsymbol{\sigma}_{n} & =\tilde{\mathbf{C}}_{n p}^{k} \boldsymbol{\varepsilon}_{p}+\tilde{\mathbf{C}}_{n n}^{k} \boldsymbol{\varepsilon}_{n}\end{aligned}$

Superscript $k \in\left[1, N_{l}\right]$ ranges over the number $N_{l}$ of cross-section sub-domains $\Omega_{e}^{k}$ (the whole cross-section is addressed by $\Omega_{e}=\cup_{k=1}^{N_{l}} \Omega_{e}^{k}$ ). Matrices $\tilde{\mathbf{C}}_{p p}^{k}, \tilde{\mathbf{C}}_{p n}^{k}, \tilde{\mathbf{C}}_{n p}^{k}$ and $\tilde{\mathbf{C}}_{n n}^{k}$ are the material stiffness matrices. Considering the general case of an anisotropic material, they are:

$$
\begin{aligned}
& \tilde{\mathbf{C}}_{p p}^{k}=\left[\begin{array}{lll}
\tilde{C}_{22}^{k} & \tilde{C}_{23}^{k} & \tilde{C}_{24}^{k} \\
\tilde{C}_{23}^{k} & \tilde{C}_{33}^{k} & \tilde{C}_{34}^{k} \\
\tilde{C}_{24}^{k} & \tilde{C}_{34}^{k} & \tilde{C}_{44}^{k}
\end{array}\right] \quad \tilde{\mathbf{C}}_{p n}^{k}=\tilde{\mathbf{C}}_{n p}^{k T}=\left[\begin{array}{lll}
\tilde{C}_{12}^{k} & \tilde{C}_{26}^{k} & \tilde{C}_{25}^{k} \\
\tilde{C}_{13}^{k} & \tilde{C}_{36}^{k} & \tilde{C}_{35}^{k} \\
\tilde{C}_{14}^{k} & \tilde{C}_{46}^{k} & \tilde{C}_{45}^{k}
\end{array}\right] \\
& \tilde{\mathbf{C}}_{n n}^{k}=\left[\begin{array}{lll}
\tilde{C}_{11}^{k} & \tilde{C}_{16}^{k} & \tilde{C}_{15}^{k} \\
\tilde{C}_{16}^{k} & \tilde{C}_{66}^{k} & \tilde{C}_{56}^{k} \\
\tilde{C}_{15}^{k} & \tilde{C}_{56}^{k} & \tilde{C}_{55}^{k}
\end{array}\right]
\end{aligned}
$$

For sake of brevity, coefficients $\tilde{C}_{i j}^{k}$ in Eq. (9) as function of the engineering material constants (Young's moduli $E_{i}$, Poisson's ratios $v_{i j}$ and shear moduli $G_{i j}$ ) and fibre orientation angles are not reported here. They can be found in Reddy [34].

\section{Hierarchical PGD beam elements}

In the framework of the proposed PGD-based unified finite element modelling, the variation of each displacement component versus the spatial coordinates is decomposed as a sum of coupled functions or "couples". Each couple is the product of functions $\mathbf{f}^{i}(y, z)$ and $\mathbf{v}^{i}(x)$ :

$$
\begin{aligned}
\mathbf{u}(x, y, z) & : \mathcal{D}_{\Omega} \times \mathcal{D}_{x} \\
(y, z, x) & \mapsto \mathbb{f}^{i}(y, z) \circ \mathbf{v}^{i}(x) \quad \text { with } i=1,2, \ldots, N_{c}
\end{aligned}
$$

$\mathbf{f}^{i}(y, z)$, which derives from the one-dimensional beam modelling, depends upon the cross-section coordinates $y$ and $z$ only. Function $\mathbf{v}^{i}(x)$ accounts for the variation versus the axial coordinate $x$. This latter, within the beam finite element modelling framework, is obtained via classical shape functions. $N_{c}$ is the number of couples. According to Einstein's notation, a repeated index is a dummy index that, unless otherwise stated, stands for summation. This notation is extensively used through the paper and it allows deriving the problem's governing equations in terms of a single "fundamental nucleo" regardless the approximation order over the cross-section $(N)$, the number of nodes per element along the beam axis $\left(N_{n}\right)$ and the number of couples in the PGD approximation. The actual governing equations due to fixed cross-section approximation order, number of nodes per element along the beam axis and number of couples are obtained straightforwardly via summation of the nucleo corresponding to each term of the expansion. In this sense, $N, N_{n}$ and $N_{c}$ are free parameters of the formulation. The operator "o" in Eq. (10) is Hadamard's element-wise vector product. It is defined as follows (see Horn [35]): $\mathbf{f}^{i} \circ \mathbf{v}^{i}: \mathbb{R}^{3} \rightarrow \mathbb{R}^{3}\left\{\begin{array}{l}f_{x}^{i} \\ f_{y}^{i} \\ f_{z}^{i}\end{array}\right\} \circ\left\{\begin{array}{l}v_{x}^{i} \\ v_{y}^{i} \\ v_{z}^{i}\end{array}\right\}=\left\{\begin{array}{l}f_{x}^{i} v_{x}^{i} \\ f_{y}^{i} v_{y}^{i} \\ f_{z}^{i} v_{z}^{i}\end{array}\right\}$

It should be noticed that, by its definition, Hadamard's operator is commutative and distributive and it can be regarded as a compact expression for the following matrix-vector product:

$\mathbf{f}^{i} \circ \mathbf{v}^{i}=\mathbf{v}^{i} \circ \mathbf{f}^{i}=\left[\begin{array}{ccc}f_{x}^{i} & 0 & 0 \\ 0 & f_{y}^{i} & 0 \\ 0 & 0 & f_{z}^{i}\end{array}\right]\left\{\begin{array}{c}v_{x}^{i} \\ v_{y}^{i} \\ v_{z}^{i}\end{array}\right\}=\left[\begin{array}{ccc}v_{x}^{i} & 0 & 0 \\ 0 & v_{y}^{i} & 0 \\ 0 & 0 & v_{z}^{i}\end{array}\right]\left\{\begin{array}{l}f_{x}^{i} \\ f_{y}^{i} \\ f_{z}^{i}\end{array}\right\}$

Although the two notations are equivalent, in the theoretical developments Hadamard's product is mainly used. The matrix notation is introduced when matrix multiplication has to be done on a term in the Hadamard product. The following approximations over the beam cross-section and its axis are introduced:

$\mathbf{f}^{i}(y, z)=F_{\tau}(y, z) \mathbf{q}_{\mathcal{D}_{\Omega} \tau}^{i} \quad$ with $\tau=1,2, \ldots, N_{u}$

$\mathbf{v}^{i}(x)=N_{j}(x) \mathbf{q}_{\mathcal{D}_{x} j}^{i} \quad$ with $j=1,2, \ldots, N_{n}$

$F_{\tau}(y, z)$ are the a priori approximating functions over the beam cross-section. Within this work, they are MacLaurin's polynomials. This choice is inspired by Euler-Bernoulli's and Timoshenko's classical beam models. The dummy index $\tau$ ranges over the number of cross-section approximation terms $N_{u}$. It depends upon the cross-section approximation order $N$ as follows:

$N_{u}=\frac{(N+1)(N+2)}{2}$

$N_{u}$ and $F_{\tau}$ as functions of $N$ can be obtained through Pascal's triangle as shown in Table $1 . N_{j}(x)$ are the classical Lagrangian finite element shape functions. They approximate the displacements along the beam axis in a $C^{0}$ sense up to an order $N_{n}-1$. The dummy index $j$ ranges over the number of nodes per element. Linear, quadratic and cubic elements along the beam axis are considered. These elements are addressed by "B2", "B3" and "B4", respectively. For the sake of simplicity, their shape functions are not reported here. They can be found in Bathe [36]. $\mathbf{q}_{\mathcal{D}_{\Omega} \tau}^{i}$ and $\mathbf{q}_{\mathcal{D}_{x} j}^{i}$ are the unknowns for the cross-section $\left(\mathcal{D}_{\Omega}\right)$ and axis $\left(\mathcal{D}_{x}\right)$ approximation spaces, respectively. By replacing Eq. (13) within Eq. (10), the displacement field becomes:

$\mathbf{u}(x, y, z)=F_{\tau}(y, z) N_{j}(x) \mathbf{q}_{\mathcal{D}_{\Omega} \tau}^{i} \circ \mathbf{q}_{\mathcal{D}_{x} j}^{i}$

Within a PGD framework, a stiffness matrix for each approximation space is derived. Thanks to the Unified Formulation, these matrices are obtained in a nuclear form via the weak form of the Principle of Virtual Displacements:

$\delta \mathcal{L}_{\text {int }}^{e}-\delta \mathcal{L}_{\text {ext }}^{e}=0$

where $\delta$ represents a virtual variation, $\mathcal{L}_{\text {int }}^{e}$ the strain energy and $\mathcal{L}_{\text {ext }}^{e}$ the work done by the external loads. Using Eq. (15), the virtual variation of the displacement reads:

$\delta \mathbf{u}^{T}=\left(\delta \mathbf{q}_{\mathcal{D}_{\Omega} \sigma}^{s T} \circ \mathbf{q}_{\mathcal{D}_{x} l}^{s}+\delta \mathbf{q}_{\mathcal{D}_{x} l}^{s T} \circ \mathbf{q}_{\mathcal{D}_{\Omega} \sigma}^{s}\right) F_{\sigma} N_{l}$

By recalling Eq. (12), Eq. (17) is rewritten using Hadamard's product matrix notation:

$\delta \mathbf{u}^{T}=\left(\delta \mathbf{q}_{\mathcal{D}_{\Omega} \sigma}^{s T} \mathbf{Q}_{\mathcal{D}_{x} l}^{s}+\delta \mathbf{q}_{\mathcal{D}_{x} l}^{s T} \mathbf{Q}_{\mathcal{D}_{\Omega} \sigma}^{s}\right) F_{\sigma} N_{l}$

where $\mathbf{Q}_{\mathcal{D}_{x} l}^{s}$ and $\mathbf{Q}_{\mathcal{D}_{\Omega} \sigma}^{s}$ are the following diagonal matrices: 
Table 1

MacLaurin's polynomials via Pascal's triangle.

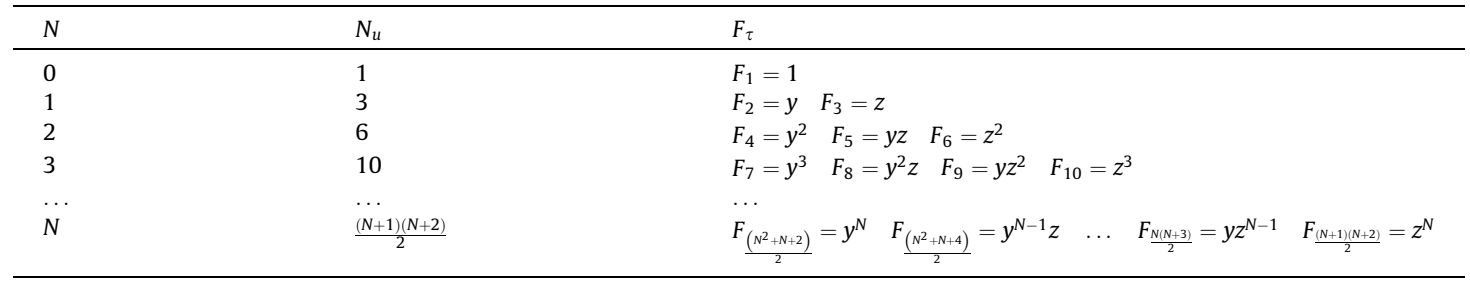

$\mathbf{Q}_{\mathcal{D}_{x} l}^{s}=\left[\begin{array}{ccc}q_{\mathcal{D}_{X} l x}^{s} & 0 & 0 \\ 0 & q_{\mathcal{D}_{x} l y}^{s} & 0 \\ 0 & 0 & q_{\mathcal{D}_{x} l z}^{s}\end{array}\right] \mathbf{Q}_{\mathcal{D}_{\Omega} \sigma}^{s}=\left[\begin{array}{ccc}q_{\mathcal{D}_{\Omega} \sigma x}^{s} & 0 & 0 \\ 0 & q_{\mathcal{D}_{\Omega} \sigma y}^{s} & 0 \\ 0 & 0 & q_{\mathcal{D}_{\Omega} \sigma z}^{s}\end{array}\right]$

\subsection{Virtual variation of the strain energy}

Coherently with the stresses and strains grouping in Eqs. (2) and (3), the virtual variation of the internal work reads:

$\delta \mathcal{L}_{\text {int }}^{e}=\int_{V_{e}}\left(\delta \boldsymbol{\varepsilon}_{p}^{T} \boldsymbol{\sigma}_{p}+\delta \boldsymbol{\varepsilon}_{n}^{T} \boldsymbol{\sigma}_{n}\right) d V$

where $V_{e}$ stands for the element volume:

$V_{e}=\Omega_{e} \times l_{e}$

being $l_{e}$ the element axial length and $\Omega_{e}$ the element cross-section. The cross-section is assumed to be constant along the beam axis. The virtual variation of the strains accounting for the displacement approximation in Eq. (15) is:

$$
\begin{aligned}
& \delta \boldsymbol{\varepsilon}_{n}=\left(\delta \mathbf{q}_{\mathcal{D}_{\Omega} \sigma}^{s T} \mathbf{Q}_{\mathcal{D}_{x} l}^{s}+\delta \mathbf{q}_{\mathcal{D}_{x}}^{s T} \mathbf{Q}_{\mathcal{D}_{\Omega} \sigma}^{s}\right)\left[N_{l}\left(\mathbf{D}_{n p} F_{\sigma} \mathbf{I}\right)^{T}+F_{\sigma} N_{l, x} \mathbf{I}\right] \\
& \delta \boldsymbol{\varepsilon}_{p}=\left(\delta \mathbf{q}_{\mathcal{D}_{\Omega} \sigma}^{s T} \mathbf{Q}_{\mathcal{D}_{x} l}^{s}+\delta \mathbf{q}_{\mathcal{D}_{x} l}^{s T} \mathbf{Q}_{\mathcal{D}_{\Omega} \sigma}^{s}\right) N_{l}\left(\mathbf{D}_{p} F_{\sigma} \mathbf{I}\right)^{T}
\end{aligned}
$$

After substitution of the constitutive equations, Eq. (8), the geometric relations, Eqs. (6) and (22), and the displacement approximation, Eq. (15), the virtual variation of the element strain energy becomes:

$\delta \mathcal{L}_{\text {int }}^{e}=\delta \mathbf{q}_{\mathcal{D}_{\Omega} \sigma}^{s T} \mathbf{K}_{\mathcal{D}_{x}}^{\sigma \tau s i} \mathbf{q}_{\mathcal{D}_{\Omega} \tau}^{i}+\delta \mathbf{q}_{\mathcal{D}_{x}}^{s T} \mathbf{K}_{\mathcal{D}_{\Omega}}^{l j s i} \mathbf{q}_{\mathcal{D}_{x} j}^{i}$

where:

$\mathbf{K}_{\mathcal{D}_{x}}^{\sigma \tau s i}=\mathbf{Q}_{\mathcal{D}_{x} l}^{s} \mathbf{K}^{l j \sigma \tau} \mathbf{Q}_{\mathcal{D}_{x} j}^{i}$

$\mathbf{K}_{\mathcal{D}_{\Omega}}^{l j s i}=\mathbf{Q}_{\mathcal{D}_{\Omega} \sigma}^{s} \mathbf{K}^{l j \sigma \tau} \mathbf{Q}_{\mathcal{D}_{\Omega} \tau}^{i}$

$\mathbf{K}_{\mathcal{D}_{x}}^{\sigma \tau s i}$ and $\mathbf{K}_{\mathcal{D}_{\Omega}}^{l j s i}$ are the projection of $\mathbf{K}^{l j \sigma \tau}$ over the space $\mathcal{D}_{x}$ and $\mathcal{D}_{\Omega}$, respectively. $\mathbf{K}^{\mathrm{lj} \sigma \tau} \in \mathbb{R}^{3 \times 3}$ is the fundamental nucleo of the element stiffness matrix as derived from a classical finite element approach (see Carrera et al. [37,19]:

$$
\begin{aligned}
\mathbf{K}^{l j \sigma \tau}= & \int_{l_{e}} \int_{\cup_{k=1}^{N_{l}} \Omega_{e}^{k}}\left\{\left[N_{l}\left(\mathbf{D}_{n p} F_{\sigma}\right)^{T}+N_{l, x} F_{\sigma} \mathbf{I}\right]\right. \\
& {\left[N_{j} \tilde{\mathbf{C}}_{n p}^{k}\left(\mathbf{D}_{p} F_{\tau}\right)+N_{j} \tilde{\mathbf{C}}_{n n}^{k}\left(\mathbf{D}_{n p} F_{\tau}\right)+N_{j, x} F_{\tau} \tilde{\mathbf{C}}_{n n}^{k}\right] } \\
& \left.+N_{l}\left(\mathbf{D}_{p} F_{\sigma}\right)^{T}\left[N_{j} \tilde{\mathbf{C}}_{p p}^{k}\left(\mathbf{D}_{p} F_{\tau}\right)+N_{j} \tilde{\mathbf{C}}_{p n}^{k}\left(\mathbf{D}_{n p} F_{\tau}\right)+N_{j, x} F_{\tau} \tilde{\mathbf{C}}_{p n}^{k}\right]\right\} d \Omega d x
\end{aligned}
$$

The explicit expression of its components is:

$$
\begin{aligned}
K_{x x}^{l j \sigma \tau}= & I_{l j}\left(J_{\sigma, y}^{66} s_{, y}+J_{\sigma, y}^{56} \tau_{, z}+J_{\sigma_{, z} \tau_{, y}}^{56}+J_{\sigma_{, z} \tau_{, z}}^{55}\right)+I_{l j, x}\left(J_{\sigma, y}^{16}+J_{\sigma_{, z} \tau}^{15}\right) \\
& +I_{l, x j}\left(J_{\sigma \tau, y}^{16}+J_{\sigma \tau, z}^{15}\right)+I_{l, x j, x} J_{\sigma \tau}^{11} \\
K_{y y}^{l j \sigma \tau}= & I_{l j}\left(J_{\sigma_{, y} \tau_{, y}}^{22}+J_{\sigma, y}^{24} \tau_{, z}+J_{\sigma_{, z} \tau_{, y}}^{24}+J_{\sigma_{, z} \tau_{, z}}^{44}\right)+I_{l j, x}\left(J_{\sigma, y}^{26}+J_{\sigma_{, z} \tau}^{46}\right) \\
& +I_{l, x j}\left(J_{\sigma \tau, y}^{26}+J_{\sigma \tau, z}^{46}\right)+I_{l, x j, x} J_{\sigma \tau}^{66}
\end{aligned}
$$

$$
\begin{aligned}
& K_{z z}^{l j \sigma \tau}=I_{l j}\left(J_{\sigma, y}^{44} \tau,+J_{\sigma, y}^{34} \tau_{, z}+J_{\sigma, z}^{34} \tau,+J_{\sigma_{, z} \tau, z}^{33}\right)+I_{l j, x}\left(J_{\sigma, y}^{45}+J_{\sigma, z}^{35} \tau\right) \\
& +I_{l, x j}\left(J_{\sigma \tau, y}^{45}+J_{\sigma \tau, z}^{35}\right)+I_{l, x j, x} J_{\sigma \tau}^{55} \\
& K_{x y}^{l j \sigma \tau}=I_{l j}\left(J_{\sigma, y}^{26} \tau_{, y}+J_{\sigma, y}^{46} \tau_{, z}+J_{\sigma, z}^{25} \tau_{, y}+J_{\sigma, z}^{45} \tau_{, z}\right)+I_{l j, x}\left(J_{\sigma, y}^{66}+J_{\sigma, z}^{56}\right) \\
& +I_{l, x j}\left(J_{\sigma \tau, y}^{12}+J_{\sigma \tau, z}^{14}\right)+I_{l, x j, x} J_{\sigma \tau}^{16} \\
& K_{y x}^{l j \sigma \tau}=I_{l j}\left(J_{\sigma, y}^{26} \tau, y+J_{\sigma, y}^{25} \tau_{, z}+J_{\sigma, z}^{46} \tau, y+J_{\sigma, z}^{45} \tau\right)+I_{l j, x}\left(J_{\sigma, y}^{12}+J_{\sigma, z}^{14}\right) \\
& +I_{l, x j}\left(J_{\sigma \tau, y}^{66}+J_{\sigma \tau, z}^{56}\right)+I_{l, x j, x} J_{\sigma \tau}^{16} \\
& K_{x z}^{l j \sigma \tau}=I_{l j}\left(J_{\sigma, y}^{46} \tau_{, y}+J_{\sigma, y}^{36} \tau_{, z}+J_{\sigma, z}^{45} \tau_{, y}+J_{\sigma, z}^{35} \tau_{, z}\right)+I_{l j, x}\left(J_{\sigma, y}^{56}+J_{\sigma, z}^{55}\right) \\
& +I_{l, x j}\left(J_{\sigma \tau, y}^{14}+J_{\sigma \tau, z}^{13}\right)+I_{l, x j, x} J_{\sigma \tau}^{15} \\
& K_{z x}^{l j \sigma \tau}=I_{l j}\left(J_{\sigma, y}^{46} \tau, y+J_{\sigma, y}^{45} \tau_{, z}+J_{\sigma, z}^{36} \tau,+J_{\sigma, z}^{35} \tau, z\right)+I_{l j, x}\left(J_{\sigma, y}^{14}+J_{\sigma, z}^{13} \tau\right) \\
& +I_{l, x j}\left(J_{\sigma \tau, y}^{56}+J_{\sigma \tau, z}^{55}\right)+I_{l, x j, x} J_{\sigma \tau}^{15} \\
& K_{y z}^{l j \sigma \tau}=I_{l j}\left(J_{\sigma_{, y} \tau_{, y}}^{24}+J_{\sigma_{, y} \tau_{, z}}^{23}+J_{\sigma_{, z} \tau_{, y}}^{44}+J_{\sigma_{, z} \tau, z}^{34}\right)+I_{l j, x}\left(J_{\sigma_{, y} \tau}^{25}+J_{\sigma_{, z} \tau}^{45}\right) \\
& +I_{l, x j}\left(J_{\sigma \tau, y}^{46}+J_{\sigma \tau, z}^{36}\right)+I_{l, x j, x} J_{\sigma \tau}^{56} \\
& K_{z y}^{l j \sigma \tau}=I_{l j}\left(J_{\sigma, y}^{24} \tau_{, y}+J_{\sigma_{, y} \tau, z}^{44}+J_{\sigma_{, z} \tau, y}^{23}+J_{\sigma_{, z} \tau, z}^{34}\right)+I_{l j, x}\left(J_{\sigma_{, y} \tau}^{46}+J_{\sigma_{, z} \tau}^{36}\right) \\
& +I_{l, x j}\left(J_{\sigma \tau, y}^{25}+J_{\sigma \tau, z}^{45}\right)+I_{l, x j, x} J_{\sigma \tau}^{56}
\end{aligned}
$$

The generic term $J_{\sigma_{(, \eta)} \tau_{(, \xi)}}^{g h}$ is a cross-section moment:

$J_{\sigma_{(, \eta \eta} \tau_{(, \xi)}}^{g h}=\int_{\cup_{k=1}^{N_{l}} \Omega_{e}^{k}} \tilde{C}_{g h}^{k} F_{\sigma_{(, \eta)}} F_{\tau_{(, \xi)}} d \Omega \quad$ with $(\eta, \xi)=\{y, z\}^{2}$

and it is a weighted sum (in the continuum) of each elemental cross-section area where the weight functions account for the spatial distribution of the geometry and the material. $I_{l_{(x)} j_{(x)}}$ is an integral along the element axis of the product of the shape functions and/or their derivatives:

$I_{l_{(x)} j_{(x)}}=\int_{l_{e}} N_{l_{(, x)}} N_{j_{(x)}} d x$

These integrals are evaluated numerically through Gauss' quadrature method. In order to correct the shear locking, a selective integration technique is used. The selected under-integrated term is $I_{l j}$ in $K_{x x}^{l j \sigma \tau}$ that is related to shear deformations $\gamma_{x y}$ and $\gamma_{x z}$.

\subsection{Virtual variation of the external work}

The beam can be loaded by concentrated forces $(\mathbf{F})$ and surface loads (p). The virtual variation of the external work reads:

$\delta \mathcal{L}_{\mathrm{ext}}^{e}=\delta \mathcal{L}_{\mathrm{ext}}^{e F}+\delta \mathcal{L}_{\mathrm{ext}}^{e p}$ 
3.2.1. Force loads

The virtual work of a generic force load:

$\mathbf{F}^{T}=\left\{\begin{array}{lll}F_{x} & F_{y} & F_{z}\end{array}\right\}$

applied on a generic point $\mathbf{x}_{F}=\left(x_{F}, y_{F}, z_{F}\right)$ is:

$\delta \mathcal{L}_{\mathrm{ext}}^{e F}=\delta \mathbf{u}^{T}\left(\mathbf{x}_{F}\right) \mathbf{F}$

By replacing Eq. (18) computed at $\mathbf{x}_{F}$ within Eq. (31), the virtual work of a point force becomes:

$\delta \mathcal{L}_{\mathrm{ext}}^{e F}=\delta \mathbf{q}_{\mathcal{D}_{\Omega} \sigma}^{s T} \mathbf{F}_{\mathcal{D}_{x} \sigma}^{s}+\delta \mathbf{q}_{\mathcal{D}_{x}}^{s T} \mathbf{F}_{\mathcal{D}_{\Omega} l}^{s}$

where $\mathbf{F}_{\mathcal{D}_{x} \sigma}^{s}$ and $\mathbf{F}_{\mathcal{D}_{\Omega} l}^{s} \in \mathbb{R}^{3}$ are force load vectors projected over $\mathcal{D}_{x}$ and $\mathcal{D}_{\Omega}$. They are variationally consistent with the kinematic approximation over the cross-section and the finite element approximation over the beam axis:

$\mathbf{F}_{\mathcal{D}_{x} \sigma}^{s}=\mathbf{q}_{\mathcal{D}_{x} l}^{s} \circ \mathbf{F}_{\sigma l}\left(\mathbf{x}_{F}\right)=\mathbf{q}_{\mathcal{D}_{x} l}^{s} \circ F_{\sigma}\left(y_{F}, z_{F}\right) N_{l}\left(x_{F}\right) \mathbf{F}$

$\mathbf{F}_{\mathcal{D}_{\Omega} l}^{s}=\mathbf{q}_{\mathcal{D}_{\Omega} \sigma}^{S} \circ \mathbf{F}_{\sigma l}\left(\mathbf{x}_{F}\right)=\mathbf{q}_{\mathcal{D}_{\Omega} \sigma}^{s} \circ F_{\sigma}\left(y_{F}, z_{F}\right) N_{l}\left(x_{F}\right) \mathbf{F}$

The term $\mathbf{F}_{\sigma l} \in \mathbb{R}^{3}$, defined as:

$\mathbf{F}_{\sigma l}=F_{\sigma}\left(y_{F}, z_{F}\right) N_{l}\left(x_{F}\right) \mathbf{F}$

is the fundamental nucleo of the force load vector as derived from the classical finite element formulation.

\subsubsection{Surface loads}

The following surface loads $\mathbf{p}_{y}(x, z)$ and $\mathbf{p}_{z}(x, y)$ are considered:

$$
\begin{array}{llll}
\mathbf{p}_{y}^{T}(x, z)=\left\{\begin{array}{llll}
p_{y x} & p_{y y} & p_{y z}
\end{array}\right\} & \forall(y, z) \in\left\{y=y_{p_{y}}, z \in\left[z_{p_{y} 1}, z_{p_{y} 2}\right]\right\} \\
\mathbf{p}_{z}^{T}(x, y)=\left\{\begin{array}{llll}
p_{z x} & p_{z y} & p_{z z}
\end{array}\right\} & \forall(y, z) \in\left\{y \in\left[y_{p_{z} 1}, y_{p_{z} 2}\right], z=z_{p_{z}}\right\}
\end{array}
$$

They act on surfaces whose normal is parallel to the $y$ - or $z$-axis and along the whole axial span of the element. The first subscript accounts for the normal of the surface the load is applied upon, whereas the second one stands for the load direction of application. The virtual variation of the external work is:

$$
\begin{aligned}
\delta \mathcal{L}_{\mathrm{ext}}^{e p}= & \int_{l_{e}}^{z_{p_{y} 2}} \int_{z_{p_{y} 1}} \delta \mathbf{u}^{T}\left(x, y_{p_{y}}, z\right) \mathbf{p}_{y}(x, z) d z d x \\
& +\int_{l_{e}}^{y_{p_{z} 2}} \int_{y_{p_{z} 1}} \delta \mathbf{u}^{T}\left(x, y, z_{p_{z}}\right) \mathbf{p}_{z}(x, y) d y d x
\end{aligned}
$$

Within the proposed framework, the virtual work of the surface loads is:

$\delta \mathcal{L}_{\mathrm{ext}}^{e p}=\delta \mathbf{q}_{\mathcal{D}_{\Omega} \sigma}^{s T}\left(\mathbf{P}_{z \mathcal{D}_{x} \sigma}^{s}+\mathbf{P}_{y \mathcal{D}_{x} \sigma}^{s}\right)+\delta \mathbf{q}_{\mathcal{D}_{x} l}^{s T}\left(\mathbf{P}_{z \mathcal{D}_{\Omega} l}^{s}+\mathbf{P}_{y \mathcal{D}_{\Omega} l}^{s}\right)$

being:

$$
\begin{aligned}
& \left(\mathbf{P}_{z \mathcal{D}_{x} \sigma}^{s}, \mathbf{P}_{y \mathcal{D}_{x} \sigma}^{s}\right)=\mathbf{q}_{\mathcal{D}_{x} l}^{s} \circ\left(\mathbf{P}_{z \sigma l}, \mathbf{P}_{y \sigma l}\right) \\
& \left(\mathbf{P}_{z \mathcal{D}_{\Omega} l}^{s}, \mathbf{P}_{y D_{\Omega} l}^{s}\right)=\mathbf{q}_{\mathcal{D}_{\Omega} \sigma}^{s} \circ\left(\mathbf{P}_{z \sigma l}, \mathbf{P}_{y \sigma l}\right)
\end{aligned}
$$

and:

$\mathbf{P}_{z \sigma l}=\int_{l_{e}} N_{l}(x) \int_{y_{p_{z} 1}}^{y_{p_{z} 2}} F_{\sigma}\left(y, z_{p_{z}}\right) \mathbf{p}_{z}(x, y) d y d x$

$\mathbf{P}_{y \sigma l}=\int_{l_{e}} N_{l}(x) \int_{z_{p_{y} 1}}^{z_{p_{y} 2}} F_{\sigma}\left(y_{p_{y}}, z\right) \mathbf{p}_{y}(x, z) d z d x$

are the classical finite element fundamental nuclei of the surface loads.

For the sake of conciseness, the following generic notation for the external loads is introduced:
$\mathbf{R}_{\mathcal{D}_{x} \sigma}^{s}=\mathbf{F}_{\mathcal{D}_{x} \sigma}^{s}+\mathbf{P}_{z D_{x} \sigma}^{s}+\mathbf{P}_{y \mathcal{D}_{x} \sigma}^{s}=\mathbf{q}_{\mathcal{D}_{x} l}^{s} \circ \mathbf{R}_{\sigma l}$

$\mathbf{R}_{\mathcal{D}_{\Omega} l}^{s}=\mathbf{F}_{\mathcal{D}_{\Omega} l}^{s}+\mathbf{P}_{z \mathcal{D}_{\Omega} l}^{s}+\mathbf{P}_{y \mathcal{D}_{\Omega} l}^{s}=\mathbf{q}_{\mathcal{D}_{\Omega} \sigma}^{s} \circ \mathbf{R}_{\sigma l}$

with:

$\mathbf{R}_{\sigma l}=\mathbf{F}_{\sigma l}+\mathbf{P}_{z \sigma l}+\mathbf{P}_{y \sigma l}$

\section{Problem formulation and solution}

\subsection{Assembling procedure at element level}

Two coupled nucleal non-linear equations are obtained by replacing the expressions of the virtual strain energy and the external works within the PVD statement:

$\delta \mathbf{q}_{\mathcal{D}_{\Omega} \sigma}^{s}: \mathbf{K}_{\mathcal{D}_{x}}^{\sigma \tau s i} \mathbf{q}_{\mathcal{D}_{\Omega} \tau}^{i}=\mathbf{R}_{\mathcal{D}_{x} \sigma}^{s}$

$\delta \mathbf{q}_{\mathcal{D}_{x} l}^{s}: \mathbf{K}_{\mathcal{D}_{\Omega}}^{l j s i} \mathbf{q}_{\mathcal{D}_{x} j}^{i}=\mathbf{R}_{\mathcal{D}_{\Omega} l}^{s}$

Accounting for Eqs. (24) and (40), Eq. (42) read:

$\left(\mathbf{Q}_{\mathcal{D}_{x} l}^{s} \mathbf{K}^{l j \sigma \tau} \mathbf{Q}_{\mathcal{D}_{x} j}^{i}\right) \mathbf{q}_{\mathcal{D}_{\Omega} \tau}^{i}=\mathbf{q}_{\mathcal{D}_{x} l}^{s} \circ \mathbf{R}_{\sigma l}$
$\left(\mathbf{Q}_{\mathcal{D}_{\Omega} \sigma}^{s} \mathbf{K}^{l j \sigma \tau} \mathbf{Q}_{\mathcal{D}_{\Omega} \tau}^{i}\right) \mathbf{q}_{\mathcal{D}_{x} j}^{i}=\mathbf{q}_{\mathcal{D}_{\Omega} \sigma}^{s} \circ \mathbf{R}_{\sigma l}$

Once the expansion order over the cross-section $N$ and the number of nodes per element $N_{n}$ are fixed, the nucleo of the problem over the cross-section has to be expanded over the indexes $\sigma$ and $\tau \in\left\{1,2, \ldots, N_{u}\right\}$ and summed over the element nodes indexes $l$ and $j \in\left\{1,2, \ldots, N_{n}\right\}$ :

$\mathbf{q}_{\mathcal{D}_{\Omega} \tau}^{i} \in \mathbb{R}^{3} \rightarrow \mathbf{q}_{\mathcal{D}_{\Omega}}^{i} \in \mathbb{R}^{3 N_{u}}$

$\mathbf{R}_{\mathcal{D}_{x} \sigma}^{s} \in \mathbb{R}^{3} \rightarrow \mathbf{R}_{\mathcal{D}_{x}}^{s} \in \mathbb{R}^{3 N_{u}}$

$\mathbf{K}_{\mathcal{D}_{x}}^{\sigma \tau s i} \in \mathbb{R}^{3 \times 3} \rightarrow \mathbf{K}_{\mathcal{D}_{x}}^{s i} \in \mathbb{R}^{3 N_{u} \times 3 N_{u}}$

The expansion procedure increases the dimension (from $\mathbb{R}^{3}$ to $\mathbb{R}^{3 N_{u}}$ ) of the expanded quantity: its nuclear form is "unfolded" over the whole approximation space. Expansion and summation are graphically presented in Fig. 2. The dimension of the problem to be solved over the cross-section domain $\left(N_{\mathcal{D}_{\Omega}}\right)$ is:

$N_{\mathcal{D}_{\Omega}}=3 N_{u}=3 \frac{(N+1)(N+2)}{2}$

The nucleo of the companion problem over the beam axis has to be expanded over the indexes $l$ and $j$ and summed over the cross-section indexes $\sigma$ and $\tau$, see Fig. 3:

$\mathbf{q}_{\mathcal{D}_{x} j}^{i} \in \mathbb{R}^{3} \rightarrow \mathbf{q}_{\mathcal{D}_{x}}^{i} \in \mathbb{R}^{3 N_{n}}$

$\mathbf{R}_{\mathcal{D}_{\Omega} l}^{s} \in \mathbb{R}^{3} \rightarrow \mathbf{R}_{\mathcal{D}_{\Omega}}^{s} \in \mathbb{R}^{3 N_{n}}$

$\mathbf{K}_{\mathcal{D}_{\Omega}}^{l j s i} \in \mathbb{R}^{3 \times 3} \rightarrow \mathbf{K}_{\mathcal{D}_{\Omega}}^{s i} \in \mathbb{R}^{3 N_{n} \times 3 N_{n}}$

After this assembling procedure, the problems in Eq. (42) become:

$\mathbf{K}_{\mathcal{D}_{x}}^{s i} \mathbf{q}_{\mathcal{D}_{\Omega}}^{i}=\mathbf{R}_{\mathcal{D}_{x}}^{s}$

$\mathbf{K}_{\mathcal{D}_{\Omega}}^{s i} \mathbf{q}_{\mathcal{D}_{x}}^{i}=\mathbf{R}_{\mathcal{D}_{\Omega}}^{s}$

and hold at element level.

\subsection{Assembling procedure at structural level}

The problem at structural level is obtained by writing the PVD statement for the whole beam:

$\delta \mathcal{L}_{\text {int }}-\delta \mathcal{L}_{\text {ext }}=\sum_{e=1}^{N_{e}}\left(\delta \mathcal{L}_{\text {int }}^{e}-\delta \mathcal{L}_{\text {ext }}^{e}\right)=0$ 


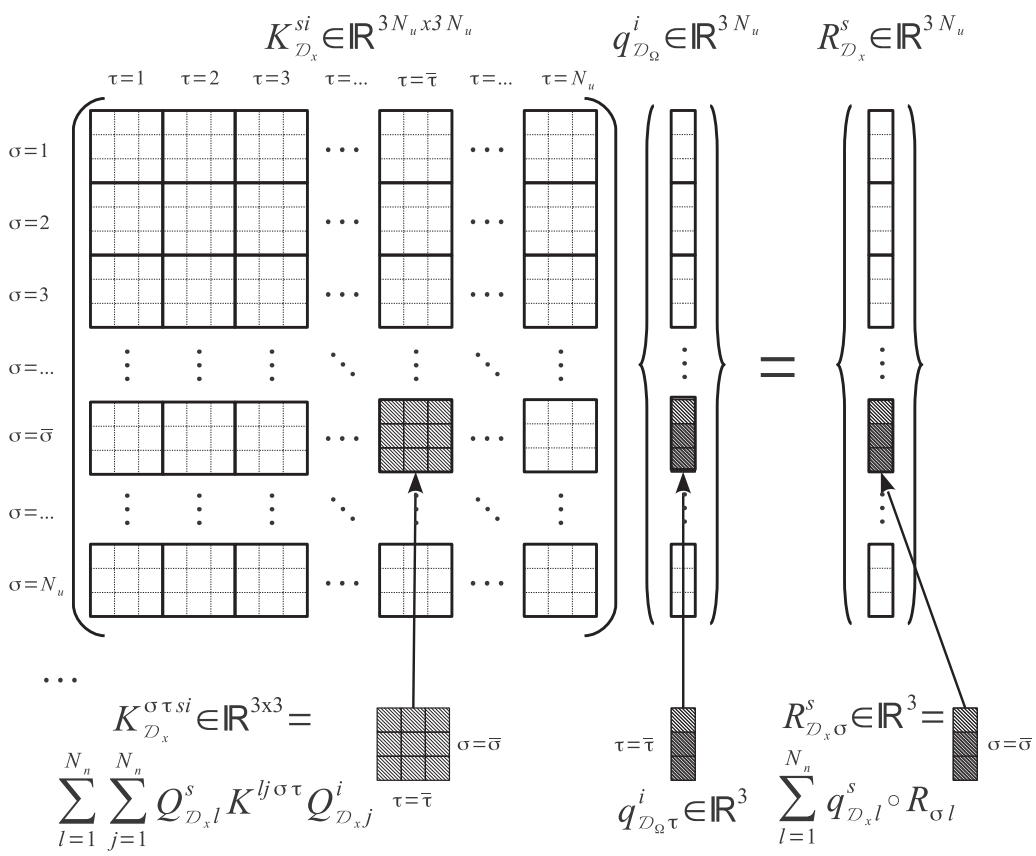

Fig. 2. Assembling procedure of the cross-section problem $\mathcal{D}_{\Omega}$ at element level.

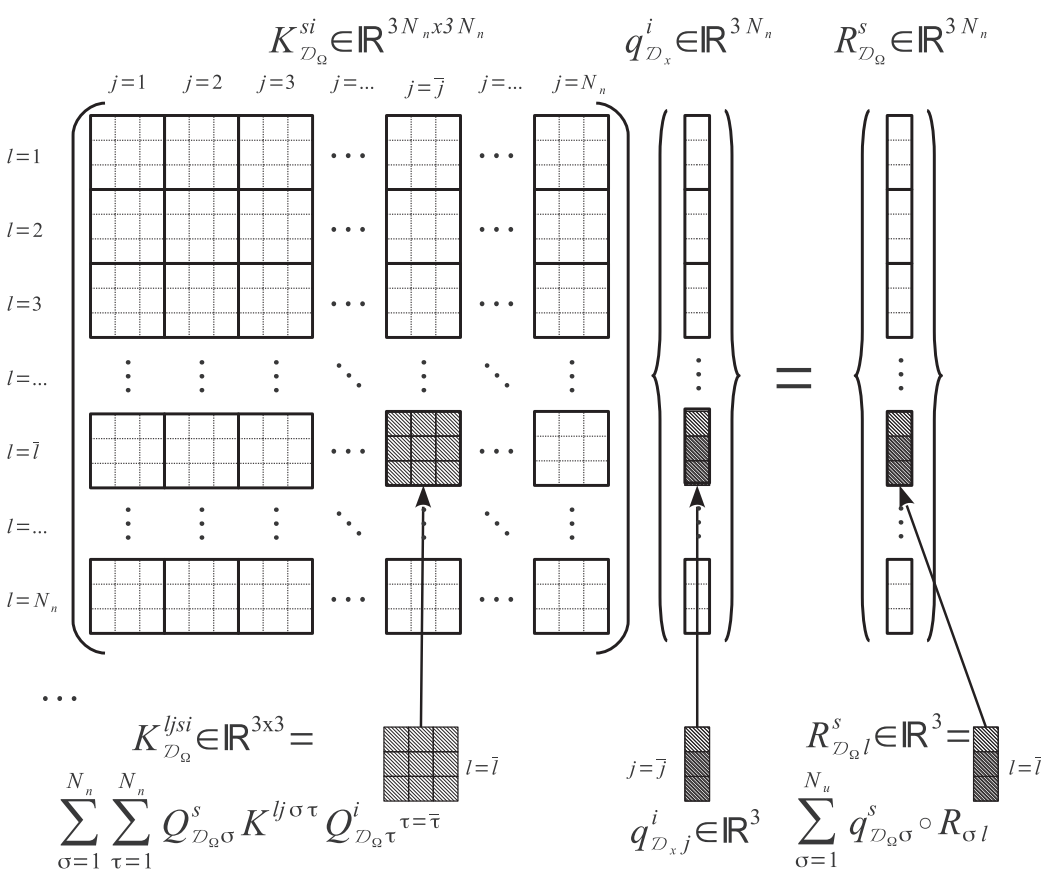

Fig. 3. Assembling procedure of the axial finite element problem $\mathcal{D}_{x}$ at element level.

where $N_{e}$ is the number of finite elements used to discretise the beam axis. The dimension of the problem to be solved over the axial domain is:

$N_{\mathcal{D}_{x}}=3\left[N_{e}\left(N_{n}-1\right)+1\right]$

It is worth underlining that the assembling procedure at structural level derives from the finite element solution method or, more generally, from a weak form solution type. Therefore, it directly affects the problem on the beam axis domain $\mathcal{D}_{x}$ only. The unknown vector $\mathbf{q}_{\mathcal{D}_{x}}^{i}$, the stiffness matrix $\mathbf{K}_{\mathcal{D}_{\Omega}}^{s i}$ and the load vector $\mathbf{F}_{\mathcal{D}_{\mathcal{O}}}^{s}$ are expanded over the discretisation domain using classical finite element assembling procedures based upon the congruency and equilibrium at a node shared by two consecutive elements, see Bathe [36]:

$$
\begin{aligned}
& \mathbf{q}_{\mathcal{D}_{x}}^{i} \in \mathbb{R}^{3 N_{n}} \rightarrow \hat{\mathbf{q}}_{\mathcal{D}_{x}}^{i} \in \mathbb{R}^{N_{\mathcal{D}_{x}}} \\
& \mathbf{R}_{\mathcal{D}_{\Omega}}^{s} \in \mathbb{R}^{3 N_{n}} \rightarrow \hat{\mathbf{R}}_{\mathcal{D}_{\Omega}}^{s} \in \mathbb{R}^{N_{\mathcal{D}_{x}}} \\
& \mathbf{K}_{\mathcal{D}_{\Omega}}^{s i} \in \mathbb{R}^{3 N_{n} \times 3 N_{n}} \rightarrow \hat{\mathbf{K}}_{\mathcal{D}_{\Omega}}^{s i} \in \mathbb{R}^{N_{\mathcal{D}_{x}} \times N_{\mathcal{D}_{x}}}
\end{aligned}
$$

The problem on $\mathcal{D}_{\Omega}$ is affected indirectly by this further assembling step because of its coupling with the problem on $\mathcal{D}_{x}$. The dimension of this latter problem, therefore, does not change and it is equal to $N_{\mathcal{D}_{\Omega}}$. The stiffness matrix $\mathbf{K}_{\mathcal{D}_{x}}^{s i}$ and the load vector 
$\mathbf{R}_{\mathcal{D}_{x}}^{s}$ of each element are simply summed. Each element contributes to the problem on the cross-section in an equivalent single layer sense:

$$
\begin{aligned}
& \mathbf{q}_{\mathcal{D}_{\Omega}}^{i} \in \mathbb{R}^{N_{\mathcal{D}_{\Omega}}} \rightarrow \hat{\mathbf{q}}_{\mathcal{D}_{\Omega}}^{i} \in \mathbb{R}^{N_{\mathcal{D}_{\Omega}}} \\
& \mathbf{R}_{\mathcal{D}_{x}}^{s} \in \mathbb{R}^{N_{\mathcal{D}_{\Omega}}} \rightarrow \hat{\mathbf{R}}_{\mathcal{D}_{x}}^{s} \in \mathbb{R}^{N_{\mathcal{D}_{\Omega}}} \\
& \mathbf{K}_{\mathcal{D}_{x}}^{s i} \in \mathbb{R}^{N_{\mathcal{D}_{\Omega}} \times N_{\mathcal{D}_{\Omega}}} \rightarrow \hat{\mathbf{K}}_{\mathcal{D}_{x}}^{s i} \in \mathbb{R}^{N_{\mathcal{D}_{\Omega}} \times N_{\mathcal{D}_{\Omega}}}
\end{aligned}
$$

Finally, the problem for the whole beam within a PGD framework reads:

$$
\begin{aligned}
& \hat{\mathbf{K}}_{\mathcal{D}_{x}}^{s i} \hat{\mathbf{q}}_{\mathcal{D}_{\Omega}}^{i}=\hat{\mathbf{R}}_{\mathcal{D}_{x}}^{s} \\
& \hat{\mathbf{K}}_{\mathcal{D}_{\Omega}}^{s i} \hat{\mathbf{q}}_{\mathcal{D}_{x}}^{i}=\hat{\mathbf{R}}_{\mathcal{D}_{\Omega}}^{s}
\end{aligned}
$$

\subsection{Solution over the number of couples}

As final step, the global problem in Eq. (52) needs to be solved for each couple. The solution procedure proposed in Ammar et al. [23] is here used. On this regard, the indexes $s$ and $i$ ranging over the number of couples $N_{c}$ play a different role than indexes $(l, j)$ and $(\sigma, \tau)$ do. These latter derive from the Unified Formulation and are used to expand the nucleo of the stiffness matrices and load vectors to obtain the corresponding global terms. The former derive from the Proper Generalised Decomposition. Matrices and vectors in Eq. (52) are not expanded over $s$ and $i$. A summation over the index $i \in\{1,2, \ldots, s\}$ is performed instead and the dimensions of the two problems over $\mathcal{D}_{\Omega}$ and $\mathcal{D}_{x}$ do not change.

In the case of an unknown couple $s$ and supposing that $\hat{\mathbf{q}}_{\mathcal{D}_{\Omega}}^{r}$ and $\hat{\mathbf{q}}_{\mathcal{D}_{x}}^{r}$ with $r \in\{1,2, \ldots, s-1\}$ have been already computed, Eq. (52) become:

$\hat{\mathbf{K}}_{\mathcal{D}_{x}}^{s s} \hat{\mathbf{q}}_{\mathcal{D}_{\Omega}}^{s}=\hat{\mathbf{R}}_{\mathcal{D}_{x}}^{s}-\hat{\mathbf{K}}_{\mathcal{D}_{x}}^{s r} \hat{\mathbf{q}}_{\mathcal{D}_{\Omega}}^{r}$
$\hat{\mathbf{K}}_{\mathcal{D}_{\Omega}}^{s s} \hat{\mathbf{q}}_{\mathcal{D}_{x}}^{s}=\hat{\mathbf{R}}_{\mathcal{D}_{\Omega}}^{s}-\hat{\mathbf{K}}_{\mathcal{D}_{\Omega}}^{s r} \hat{\mathbf{q}}_{\mathcal{D}_{x}}^{r}$

The second term in each right hand side of the previous equations is summed over $r \in\{1,2, \ldots, s-1\}$ and it represents a residual term. The solution of Eq. (53) is obtained through a row-wise scheme: the residuals account for the interaction between the couple currently computed with those already determined In this sense, each new couple represents a solution refinement. The matrices $\hat{\mathbf{K}}_{D_{x}}^{s r}$ and $\hat{\mathbf{K}}_{\mathcal{D}_{\Omega}}^{s r}$ are not, in general, symmetric because they are computed using the unknown vectors of different couples $(r \neq s)$. As far as the left hand side terms of Eq. (53) are concerned, Einstein's notation does

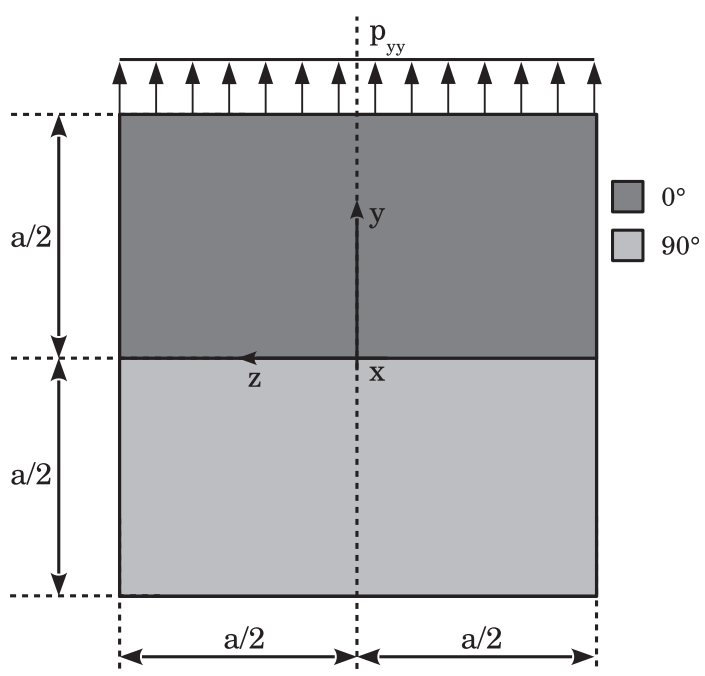

Fig. 4. Square cross-section geometry and surface bending load. not apply for $s$ since it is repeated more than twice. A fixed point method is, then, used to solve the coupled non-linear problem in Eq. (53):

1. a tentative solution $\hat{\mathbf{q}}_{\mathcal{D}_{x}}^{s_{0}}$ is first set for the problem on $\mathcal{D}_{\Omega}$ and $\hat{\mathbf{q}}_{\mathcal{D}_{\Omega}}^{s_{0}}$ is obtained,

2. $\hat{\mathbf{q}}_{\mathcal{D}_{\Omega}}^{s_{\Omega}}$ is, then, used for the problem on $\mathcal{D}_{x}$ and $\hat{\mathbf{q}}_{D_{x}}^{s_{1}}$ is computed,

3. the procedure at the previous two points is iterated until the following convergence criterion is satisfied:

$$
\max \left(\frac{\left\|\hat{q}_{\mathcal{D}_{x}}^{s_{m}}-\hat{q}_{\mathcal{D}_{x}}^{s_{m-1}}\right\|_{2}}{\left\|\hat{q}_{\mathcal{D}_{x}}^{s_{m-1}}\right\|_{2}}, \frac{\left\|\hat{q}_{\mathcal{D}_{\Omega}}^{s_{m}}-\hat{q}_{\mathcal{D}_{\Omega}}^{s_{m-1}}\right\|_{2}}{\left\|\hat{q}_{\mathcal{D}_{\Omega}}^{s_{m-1}}\right\|_{2}}\right) \leqslant \varepsilon
$$

being $\varepsilon=10^{-6}$ and $\|\square\|_{2}$ the Euclidean norm.

For the considered numerical investigation, it has been observed that the number of iteration required to reach the convergence increases for each successive couple. Nevertheless, about 15 iterations have been required, at worst. At each iteration, two problems of dimension $N_{\mathcal{D}_{\Omega}}$ and $N_{\mathcal{D}_{x}}$ are solved. It should be noticed that the order of an equivalent classical finite element problem is $3 \cdot \frac{(N+1)(N+2)}{2} \cdot\left[N_{e}\left(N_{n}-1\right)+1\right]$ or, equivalently, $\frac{N_{\mathcal{D}_{\Omega}} \cdot N_{\mathcal{D}_{X}}}{3}$.

\subsection{Boundary conditions}

As far as Dirichlet boundary conditions are concerned, the case of nil prescribed displacements along the beam axis is considered. The same principle as for the assembling procedure at structural level is valid. A displacements' constrain is directly imposed for the problem on the axial space $\mathcal{D}_{x}$ :

$u_{\varphi}\left(x_{\underline{j}}, y, z\right)=F_{\tau}(y, z) N_{\underline{j}}\left(x_{\underline{j}}\right) q_{\varphi \mathcal{D}_{\Omega} \tau}^{i} q_{\varphi \mathcal{D}_{x} \underline{j}}^{i}=0$

where subscript $\varphi$ stands for a generic spatial coordinate, $j$ for the constrained node index and $x_{j}$ for its axial position. Summation over the reaped index $j$ is not actually performed because of the well known properties of the shape functions and Eq. (55) reduces to:

$q_{\varphi \mathcal{D}_{x} j}^{i}=0$

A numerical penalisation technique is used for imposing a nil displacement: the diagonal term of the stiffness matrix $\hat{\mathbf{K}}_{\mathcal{D}_{\Omega}}^{s i}$ corresponding to the constrained degree of freedom is set to a value two or three order of magnitude higher than the maximum absolute value of the matrix, see Bathe [36].

For the problem on the cross-section domain, the boundary conditions are imposed indirectly during the projection of the stiffness matrix. According to Eq. (24)a and (51), a constrained degree of freedom does not yield any contribution in the assembling procedure over the whole structure being its value is equal to zero.

\section{Numerical results and discussion}

Analyses are carried out for slender and short beams, $l / a=100$ and 10 , respectively. Square cross-sections with sides' length $a=b=1 \mathrm{~m}$ are considered. The cross-section can be prismatic or channelled (box cross-section). A [0/90] lamination is considered. Plies have equal thickness and angles are measured towards the $x$-axis. Layers are all made from an unidirectional carbon-fibre/epoxy composite material whose equivalent properties are: $E_{L} / E_{T}=25, \quad G_{T T} / E_{T}=0.2, \quad G_{L T} / E_{T}=0.5 \quad$ and $v_{L T}=v_{T T}=0.25, E_{i}$ and $G_{i j}$ stand for Young's and shear moduli, respectively, and $v_{i j}$ are the Poisson ratios. Subscripts ' $L$ ' and ' $T$ ' stand for a direction parallel or perpendicular to the fibres. 


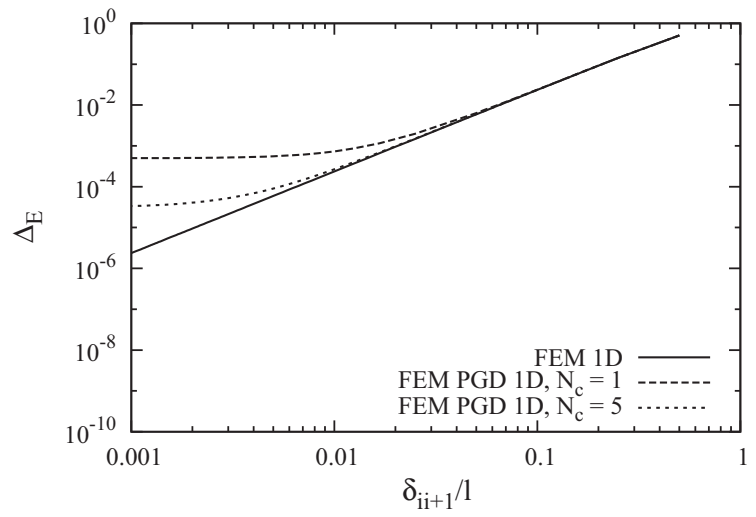

(a)

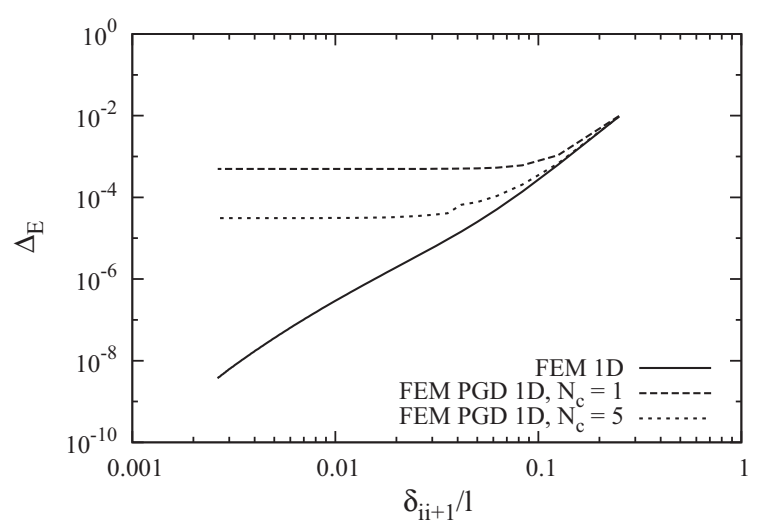

(b)

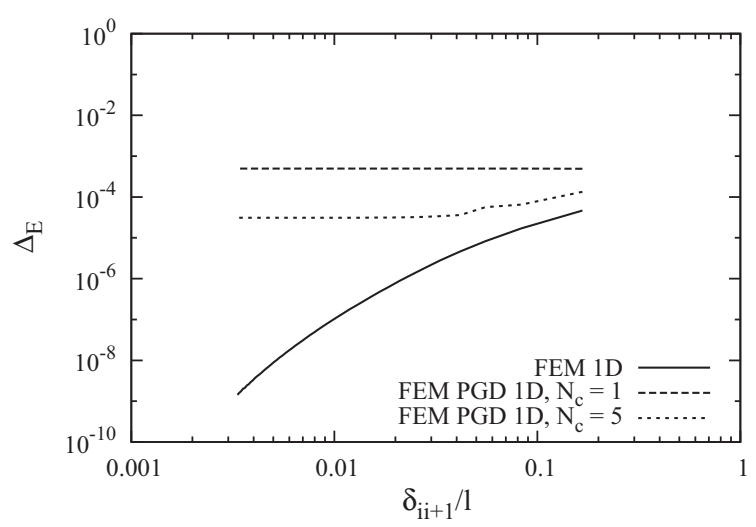

(c)

Fig. 5. Strain energy relative error $\Delta_{E}$ versus the normalised distance $\delta_{i i+1} / l$ between two consecutive nodes for (a) linear, (b) quadratic and (c) cubic element. Simply supported beam, $l / a=10$ and $N=4$.

Beams are subjected to a uniformly distributed surface load of amplitude $p_{y y}=1 \mathrm{~Pa}$ and acting on a whole cross-section side or on part of it. A simply supported configuration is considered for which a closed form, Navier-type solution implementing the same kinematics presented in this article is also present, see Giunta et al. [20]. It is also presented because, within the theory approximation, it is an exact solution of governing differential equation obtained from a strong form formulation of the PVD. The commercial code ANSYS is also used for obtaining three-dimensional finite element solutions for validation purposes. The quadratic 20-node element "Solid186" is used, see ANSYS theory manual [38]. A coarse and a fine mesh solution (addressed as "FEM 3D-C" and "FEM 3D-R", respectively) are considered to present the convergence of the three-dimensional numerical reference solution. Displacements and stresses are put into the following dimensionless form:

$$
\begin{aligned}
& \bar{u}_{i}=\frac{E_{T}}{p_{y y} a} u_{i} \text { with } i=x, z \quad \bar{u}_{y}=100 \frac{E_{T} a^{3}}{p_{y y} l^{4}} u_{z} \\
& \bar{\sigma}_{i j}=\frac{1}{p_{y y}} \sigma_{i j} \text { with } i=x, y, z
\end{aligned}
$$

\subsection{Square prismatic cross-section beam}

Square prismatic cross-section beams are first investigated. Cross-section geometry, lamination and load are shown in Fig. 4. Fig. 5 presents a convergence analysis in terms of normalised difference $\left(\Delta_{E}\right)$ in strain energy $\left(\mathcal{L}_{\text {int }}\right)$ :

$\Delta_{E}=\frac{\mathcal{L}_{\text {int }}^{\mathrm{Nav}}-\mathcal{L}_{\text {int }}}{\mathcal{L}_{\text {int }}^{\mathrm{Nav}}}$

of both FEM and FEM PGD solutions versus the number of elements along the beam axis. Superscript "Nav" in Eq. (58) stands for the Navier-type solution. Short beams are only considered, the case of slender beams being very similar. Results are obtained by a fourth-order model (nevertheless, the same conclusions apply to a generic cross-section expansion order). $\Delta_{E}$ is plotted versus the distance between two consecutive nodes $\delta_{i i+1}$ normalised versus the beam length $l$. Linear, quadratic and cubic elements are used. For the classical finite element solution, the error in the strain energy decreases as the number of nodes increases and, for a fixed number of nodes, the higher the order of the finite element shape functions, the smaller the error. As far as the PGD is concerned, the error presents an horizontal asymptote. The order of magnitude of the error is $10^{-4}$ and $10^{-5}$ for $N_{c}$ equal to one and five, regardless the element type. Unless differently stated, the results further presented are computed using 121 nodes that corresponds to 120 B2 elements, 60 B3 ones and 40 B4 elements. Fig. 6 outlines the convergence of the strain energy versus the number of couples for a slender beam and via a second-order model. Classical FEM solutions (solid lines) are reported for the sake of comparison. The FEM PGD solution converges to the classical finite element one for all the considered element types for a suitable number of couples $\left(N_{c} \geqslant 8\right)$. Tabular results show that displacements are accurately obtained by three couples, whereas five (slender beams) or six (short beams) couples are used for the considered stresses converging up to four significant digits.

Shear locking investigation is presented in Fig. 7, where the following normalised transverse displacement:

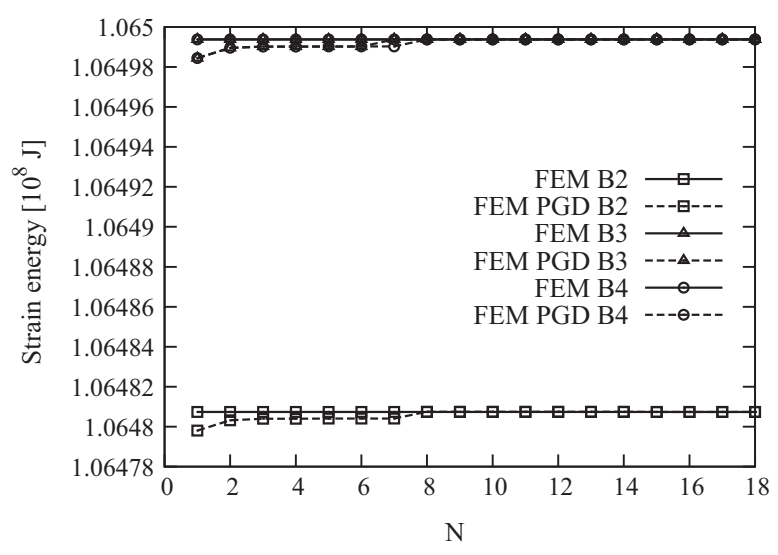

Fig. 6. Strain energy convergence versus the number of couples, B2, B3 and B4 elements, 121 nodes, $l / a=100$ and $N=2$. 


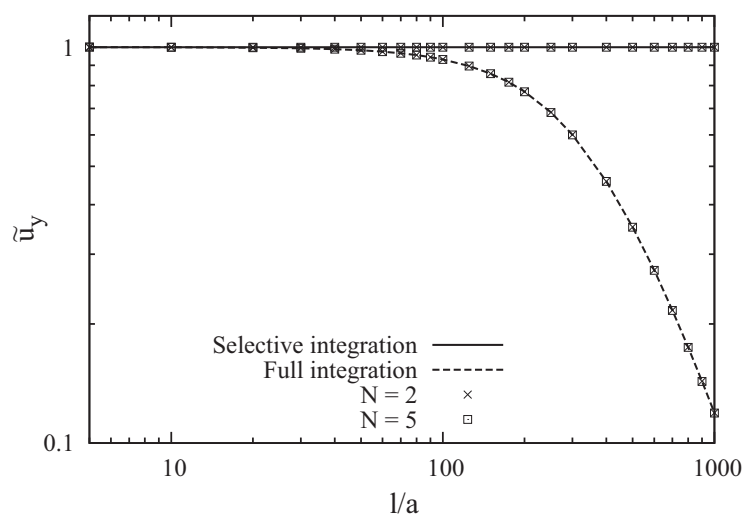

Fig. 7. Transverse displacement ratio $\tilde{u}_{y}=u_{y}(l / 2,0,0) / u_{y}^{\text {Nav }}(l / 2,0,0)$ versus $l / a$ via linear elements, $N=2$ and 5. The figure is the same for the FEM as well as FEM PGD solution regardless the value of $N_{c}$.

Table 2

Dimensionless displacements for slender and short simply supported beams under a surface bending load via three-dimensional finite element and Navier-type solutions.

\begin{tabular}{|c|c|c|c|c|c|c|}
\hline & \multicolumn{3}{|l|}{$l / a=100$} & \multicolumn{3}{|l|}{$l / a=10$} \\
\hline & $10^{-4} \times \bar{u}_{x}$ & $\bar{u}_{y}$ & $10^{-2} \times \bar{u}_{z}$ & $10^{-1} \times \bar{u}_{x}$ & $\bar{u}_{y}$ & $\bar{u}_{z}$ \\
\hline FEM 3D-R & 7.792 & 3.334 & 2.921 & 8.304 & 3.724 & 3.054 \\
\hline FEM 3D-C ${ }^{b}$ & 7.793 & 3.334 & 2.921 & 8.304 & 3.724 & 3.054 \\
\hline$N=11,12$ & 7.790 & 3.333 & 2.918 & 8.301 & 3.723 & 3.050 \\
\hline$N=9$ & 7.789 & 3.333 & 2.917 & 8.300 & 3.722 & 3.048 \\
\hline$N=7$ & 7.788 & 3.332 & 2.919 & 8.297 & 3.721 & 3.049 \\
\hline$N=6$ & 7.787 & 3.332 & 2.915 & 8.290 & 3.719 & 3.042 \\
\hline$N=4$ & 7.786 & 3.331 & 2.917 & 8.255 & 3.706 & 3.032 \\
\hline$N=3$ & 7.781 & 3.330 & 2.903 & 8.144 & 3.662 & 2.975 \\
\hline$N=2$ & 7.778 & 3.328 & 2.972 & 8.121 & 3.646 & 3.032 \\
\hline
\end{tabular}

Refined mesh: $40 \times 40 \times 400$ for $l / a=100$ and $80 \times 80 \times 80$ for $l / a=10$.

b Coarse mesh: $12 \times 12 \times 120$ for $l / a=100$ and $12 \times 12 \times 12$ for $l / a=10$.

Table 3

Dimensionless displacements for a slender simply supported beam under a surface bending load via FEM 1D and FEM PGD 1D solutions, 121 nodes and $N_{c}=3$.

\begin{tabular}{|c|c|c|c|c|c|c|}
\hline & \multicolumn{2}{|c|}{$10^{-4} \times \bar{u}_{x}$} & \multicolumn{2}{|l|}{$\bar{u}_{y}$} & \multicolumn{2}{|c|}{$10^{-2} \times \bar{u}_{z}$} \\
\hline & B2 & B3, B4 & B2 & B3, B4 & B2 & B3, B4 \\
\hline \multicolumn{7}{|l|}{ FEM 1D } \\
\hline$N=11,12$ & 7.789 & 7.790 & 3.332 & 3.333 & 2.918 & 2.918 \\
\hline$N=9$ & 7.789 & 7.789 & 3.332 & 3.333 & 2.917 & 2.917 \\
\hline$N=7$ & 7.788 & 7.788 & 3.332 & 3.332 & 2.919 & 2.919 \\
\hline$N=6$ & 7.787 & 7.787 & 3.332 & 3.332 & 2.915 & 2.915 \\
\hline$N=4$ & 7.785 & 7.786 & 3.331 & 3.331 & 2.917 & 2.917 \\
\hline$N=3$ & 7.781 & 7.781 & 3.329 & 3.330 & 2.902 & 2.903 \\
\hline$N=2$ & 7.778 & 7.778 & 3.328 & 3.328 & 2.971 & 2.972 \\
\hline \multicolumn{7}{|l|}{ FEM PGD $1 D$} \\
\hline$N=11,12$ & 7.789 & 7.790 & 3.332 & 3.333 & 2.918 & 2.918 \\
\hline$N=9$ & 7.788 & 7.789 & 3.332 & 3.333 & 2.916 & 2.917 \\
\hline$N=7$ & 7.788 & 7.788 & 3.332 & 3.332 & 2.919 & 2.919 \\
\hline$N=6$ & 7.787 & 7.787 & 3.332 & 3.332 & 2.915 & 2.915 \\
\hline$N=4$ & 7.785 & 7.786 & 3.331 & 3.331 & 2.916 & 2.917 \\
\hline$N=3$ & 7.781 & 7.781 & 3.329 & 3.330 & 2.902 & 2.903 \\
\hline$N=2$ & 7.778 & 7.778 & 3.328 & 3.328 & 2.971 & 2.972 \\
\hline
\end{tabular}

$\tilde{u}_{y}=\frac{u_{y}}{u_{y}^{\mathrm{Nav}}}$

evaluated at $(l / 2,0,0)$ is depicted versus the length-to-side ratio. Results obtained via second- and fifth-order theories are presented in the case of B2 elements (the most affected by shear locking). Full and selective integration strategies are compared. In the former case, locking appears for $l / a \geqslant 40$ whereas the latter is locking free. Results are the same regardless the solution framework (classical
Table 4

Dimensionless displacements for a short simply supported beam under a surface bending load via FEM 1D and FEM PGD 1D solutions, 121 nodes and $N_{c}=5$.

\begin{tabular}{|c|c|c|c|c|c|c|}
\hline & \multicolumn{2}{|c|}{$10^{-1} \times \bar{u}_{x}$} & \multicolumn{2}{|l|}{$\bar{u}_{y}$} & \multicolumn{2}{|l|}{$\bar{u}_{z}$} \\
\hline & B2 & B3, B4 & B2 & B3, B4 & B2 & B3, B4 \\
\hline \multicolumn{7}{|l|}{ FEM 1D } \\
\hline$N=11,12$ & 8.301 & 8.301 & 3.722 & 3.723 & 3.050 & 3.050 \\
\hline$N=9$ & 8.300 & 8.300 & 3.722 & 3.722 & 3.048 & 3.048 \\
\hline$N=7$ & 8.296 & 8.297 & 3.721 & 3.721 & 3.049 & 3.049 \\
\hline$N=6$ & 8.289 & 8.290 & 3.719 & 3.719 & 3.042 & 3.042 \\
\hline$N=4$ & 8.255 & 8.255 & 3.706 & 3.706 & 3.032 & 3.032 \\
\hline$N=3$ & 8.143 & 8.144 & 3.662 & 3.662 & 2.975 & 2.975 \\
\hline$N=2$ & 8.120 & 8.121 & 3.646 & 3.646 & 3.032 & 3.032 \\
\hline \multicolumn{7}{|l|}{ FEM PGD $1 D$} \\
\hline$N=11,12$ & 8.301 & 8.301 & 3.722 & 3.723 & 3.050 & 3.050 \\
\hline$N=9$ & 8.299 & 8.300 & 3.722 & 3.722 & 3.048 & 3.048 \\
\hline$N=7$ & 8.296 & 8.297 & 3.721 & 3.721 & 3.049 & 3.049 \\
\hline$N=6$ & 8.289 & 8.290 & 3.718 & 3.719 & 3.042 & 3.042 \\
\hline$N=4$ & 8.254 & 8.255 & 3.706 & 3.706 & 3.032 & 3.032 \\
\hline$N=3$ & 8.143 & 8.143 & 3.662 & 3.662 & 2.975 & 2.975 \\
\hline$N=2$ & 8.120 & 8.120 & 3.646 & 3.646 & 3.032 & 3.032 \\
\hline
\end{tabular}

Table 5

Dimensionless stresses for slender and short simply supported beams under a surface bending load via three-dimensional finite element and Navier-type solutions.

\begin{tabular}{|c|c|c|c|c|c|}
\hline & \multicolumn{2}{|l|}{$l / a=100$} & \multicolumn{3}{|c|}{$l / a=10$} \\
\hline & $10^{-4} \times \bar{\sigma}_{x x}$ & $\bar{\sigma}_{x y}$ & $\bar{\sigma}_{x x}$ & $10 \times \bar{\sigma}_{y y}$ & $\bar{\sigma}_{x y}$ \\
\hline FEM 3D-R ${ }^{a}$ & 2.152 & 113.8 & 216.6 & 6.354 & 10.76 \\
\hline FEM 3D-C ${ }^{b}$ & 2.152 & 115.5 & 217.2 & 6.357 & 10.98 \\
\hline$N=12$ & 2.153 & 113.7 & 216.7 & 6.381 & 10.77 \\
\hline$N=11$ & 2.152 & 113.8 & 216.6 & 6.717 & 10.79 \\
\hline$N=10$ & 2.150 & 115.4 & 216.4 & 8.557 & 10.93 \\
\hline$N=8$ & 2.153 & 115.6 & 216.6 & 9.123 & 10.91 \\
\hline$N=6$ & 2.150 & 111.0 & 216.3 & 7.102 & 10.54 \\
\hline$N=4$ & 2.151 & 100.4 & 216.2 & 3.016 & 9.557 \\
\hline$N=3$ & 2.153 & 76.28 & 213.9 & 1.514 & 7.495 \\
\hline$N=2$ & 2.154 & 73.10 & 211.4 & 2.228 & 7.215 \\
\hline
\end{tabular}

a Refined mesh: $40 \times 40 \times 400$ for $l / a=100$ and $80 \times 80 \times 80$ for $l / a=10$.

b Coarse mesh: $12 \times 12 \times 120$ for $l / a=100$ and $12 \times 12 \times 12$ for $l / a=10$.

Table 6

Dimensionless stresses for a slender simply supported beam under a surface bending load via FEM 1D and FEM PGD 1D solutions, 121 nodes and $N_{c}=5$.

\begin{tabular}{|c|c|c|c|c|c|c|}
\hline & \multicolumn{3}{|c|}{$10^{-4} \times \bar{\sigma}_{x x}$} & \multicolumn{3}{|l|}{$\bar{\sigma}_{x y}$} \\
\hline & B2 & B3 & B4 & B2 & B3 & B4 \\
\hline \multicolumn{7}{|l|}{ FEM 1D } \\
\hline$N=12$ & 2.153 & 2.153 & 2.153 & 102.6 & 128.8 & 114.4 \\
\hline$N=11$ & 2.152 & 2.153 & 2.152 & 102.7 & 128.9 & 114.5 \\
\hline$N=10$ & 2.150 & 2.150 & 2.150 & 104.3 & 130.5 & 116.1 \\
\hline$N=8$ & 2.152 & 2.153 & 2.153 & 104.1 & 130.3 & 115.8 \\
\hline$N=6$ & 2.150 & 2.151 & 2.150 & 99.48 & 125.6 & 111.2 \\
\hline$N=4$ & 2.151 & 2.151 & 2.151 & 88.92 & 115.1 & 100.7 \\
\hline$N=3$ & 2.153 & 2.153 & 2.153 & 64.83 & 90.91 & 76.53 \\
\hline$N=2$ & 2.153 & 2.154 & 2.154 & 61.67 & 87.71 & 73.33 \\
\hline \multicolumn{7}{|c|}{ FEM PGD 1D } \\
\hline$N=12$ & 2.153 & 2.153 & 2.153 & 102.7 & 128.8 & 114.4 \\
\hline$N=11$ & 2.152 & 2.153 & 2.152 & 102.8 & 128.9 & 114.5 \\
\hline$N=10$ & 2.150 & 2.151 & 2.150 & 104.4 & 130.5 & 116.1 \\
\hline$N=8$ & 2.152 & 2.153 & 2.153 & 104.1 & 130.3 & 115.9 \\
\hline$N=6$ & 2.150 & 2.151 & 2.150 & 99.50 & 125.7 & 111.3 \\
\hline$N=4$ & 2.151 & 2.151 & 2.151 & 88.92 & 115.1 & 100.7 \\
\hline$N=3$ & 2.153 & 2.153 & 2.153 & 64.84 & 90.92 & 76.54 \\
\hline$N=2$ & 2.153 & 2.154 & 2.154 & 61.66 & 87.68 & 73.32 \\
\hline
\end{tabular}

FEM or FEM with separation of the approximation space with a generic number of couples) and the kinematic approximation over the cross-section. This is due to the fact that shear locking is a 
Table 7

Dimensionless stresses for a short simply supported beam under a surface bending load via FEM 1D and FEM PGD 1D solutions, 121 nodes and $N_{c}=6$.

\begin{tabular}{|c|c|c|c|c|c|c|c|c|c|}
\hline & \multicolumn{3}{|l|}{$\bar{\sigma}_{x x}$} & \multicolumn{3}{|c|}{$\underline{10 \times \bar{\sigma}_{y y}}$} & \multicolumn{3}{|l|}{$\bar{\sigma}_{x y}$} \\
\hline & B2 & B3 & B4 & B2 & B3 & B4 & B2 & B3 & B4 \\
\hline \multicolumn{10}{|l|}{ FEM 1D } \\
\hline$N=12$ & 216.7 & 216.7 & 216.7 & 6.385 & 6.386 & 6.385 & 10.75 & 10.82 & 10.79 \\
\hline$N=11$ & 216.6 & 216.7 & 216.6 & 6.717 & 6.718 & 6.717 & 10.76 & 10.83 & 10.80 \\
\hline$N=10$ & 216.4 & 216.4 & 216.4 & 8.556 & 8.557 & 8.557 & 10.89 & 10.97 & 10.94 \\
\hline$N=8$ & 216.6 & 216.7 & 216.6 & 9.123 & 9.123 & 9.123 & 10.86 & 10.94 & 10.91 \\
\hline$N=6$ & 216.3 & 216.3 & 216.3 & 7.102 & 7.103 & 7.102 & 10.51 & 10.58 & 10.55 \\
\hline$N=4$ & 216.2 & 216.3 & 216.2 & 3.016 & 3.017 & 3.016 & 9.558 & 9.621 & 9.588 \\
\hline$N=3$ & 213.9 & 214.0 & 213.9 & 1.514 & 1.514 & 1.514 & 7.453 & 7.535 & 7.518 \\
\hline$N=2$ & 211.4 & 211.5 & 211.4 & 2.228 & 2.228 & 2.228 & 7.137 & 7.233 & 7.229 \\
\hline \multicolumn{10}{|c|}{ FEM PGD 1D } \\
\hline$N=12$ & 216.7 & 216.7 & 216.7 & 6.395 & 6.395 & 6.393 & 10.74 & 10.82 & 10.79 \\
\hline$N=11$ & 216.6 & 216.7 & 216.6 & 6.727 & 6.727 & 6.725 & 10.75 & 10.83 & 10.80 \\
\hline$N=10$ & 216.4 & 216.4 & 216.4 & 8.572 & 8.572 & 8.570 & 10.89 & 10.96 & 10.94 \\
\hline$N=8$ & 216.6 & 216.7 & 216.7 & 9.145 & 9.145 & 9.144 & 10.86 & 10.93 & 10.91 \\
\hline$N=6$ & 216.3 & 216.3 & 216.3 & 7.123 & 7.123 & 7.123 & 10.51 & 10.58 & 10.56 \\
\hline$N=4$ & 216.2 & 216.3 & 216.2 & 3.025 & 3.026 & 3.027 & 9.560 & 9.629 & 9.602 \\
\hline$N=3$ & 213.9 & 214.0 & 213.9 & 1.514 & 1.516 & 1.516 & 7.453 & 7.541 & 7.525 \\
\hline$N=2$ & 211.4 & 211.5 & 211.4 & 2.230 & 2.230 & 2.230 & 7.134 & 7.231 & 7.227 \\
\hline
\end{tabular}

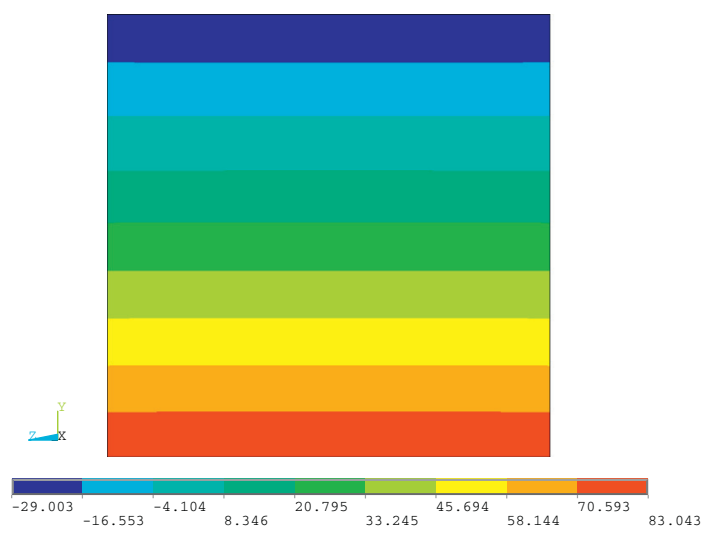

(a)

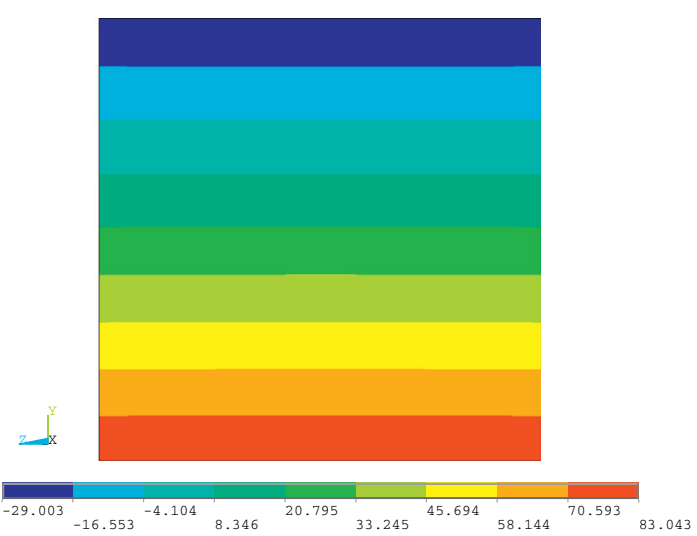

(b)

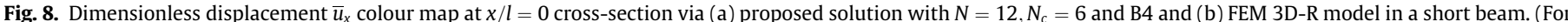
interpretation of the references to colour in this figure legend, the reader is referred to the web version of this article.)

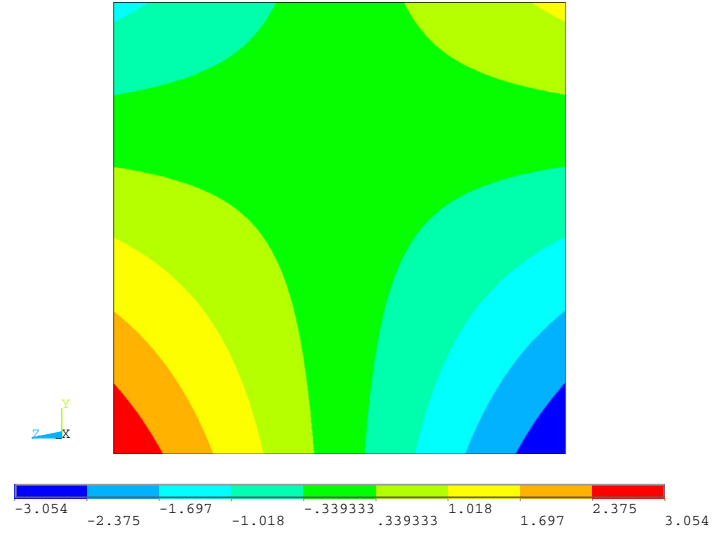

(a)

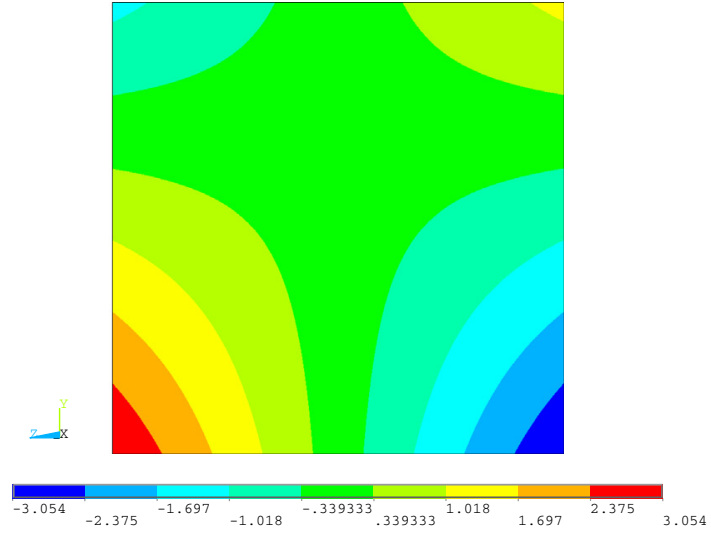

(b)

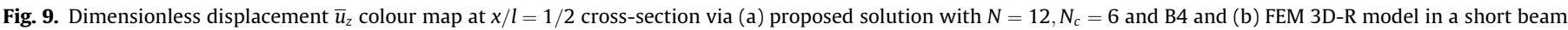
(For interpretation of the references to colour in this figure legend, the reader is referred to the web version of this article.)

numerical problem that arises as soon as a finite element approximation is used.

The dimensionless displacement in Eq. (57) are presented in Tables $2-4$ where $\bar{u}_{x}$ is evaluated at $(0,-a / 2,0), \bar{u}_{y}$ at $(l / 2,0,0)$ and $\bar{u}_{z}$ at $(l / 2,-a / 2, b / 2)$. Table 2 addresses the reference results obtained by the three-dimensional finite element model and the Navier-type solution for both slender and short beams. The three-dimensional finite element solution provides a validation 


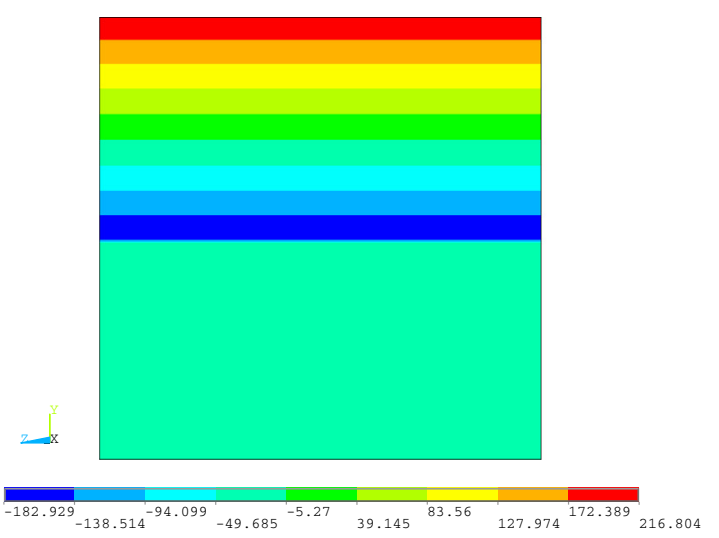

(a)

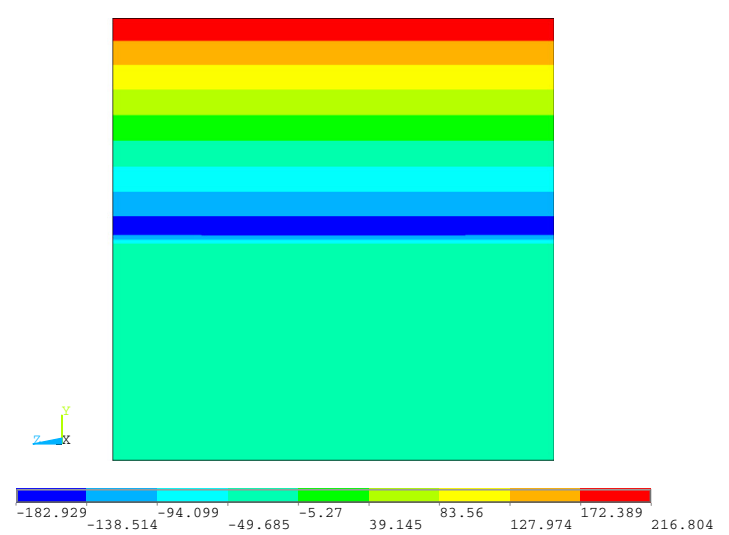

(b)

Fig. 10. Dimensionless stress $\bar{\sigma}_{x x}$ colour map at $x / l=1 / 2$ cross-section via (a) proposed solution with $N=12, N_{c}=6$ and B4 and (b) FEM 3D-R model in a short beam. (For interpretation of the references to colour in this figure legend, the reader is referred to the web version of this article.)

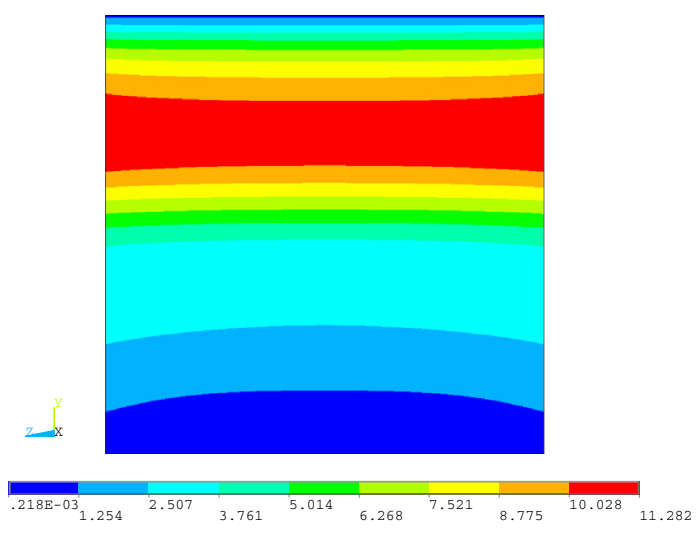

(a)

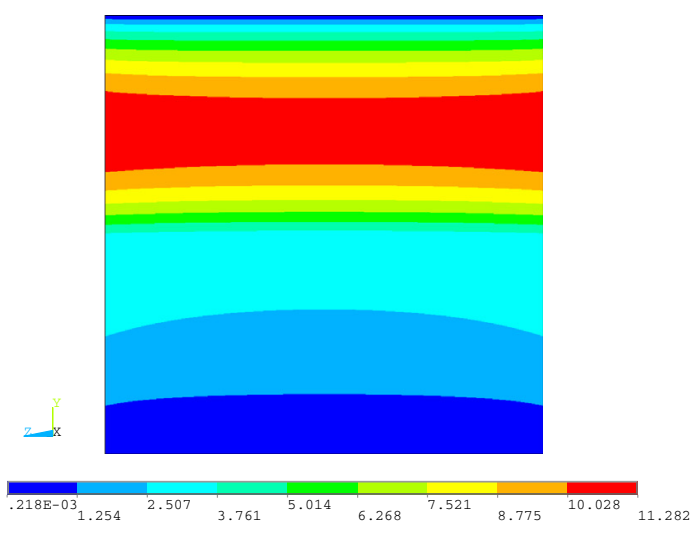

(b)

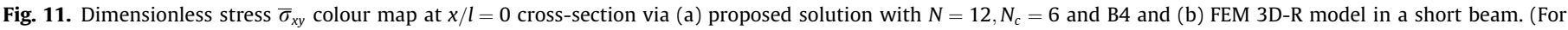
interpretation of the references to colour in this figure legend, the reader is referred to the web version of this article.)

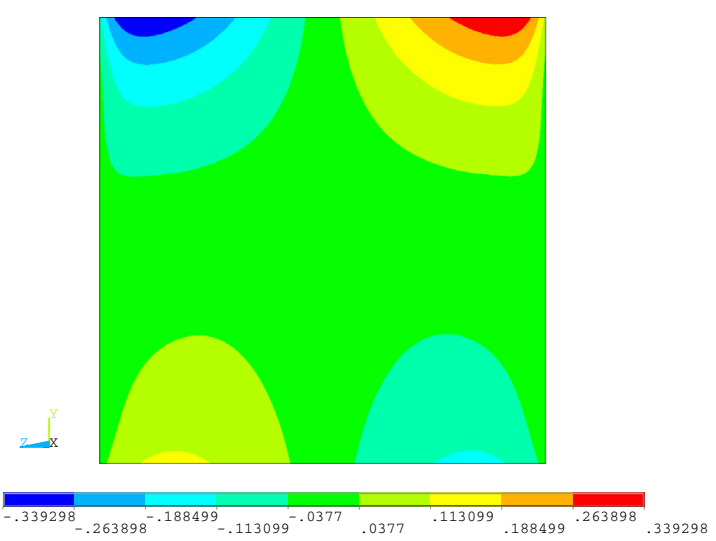

(a)

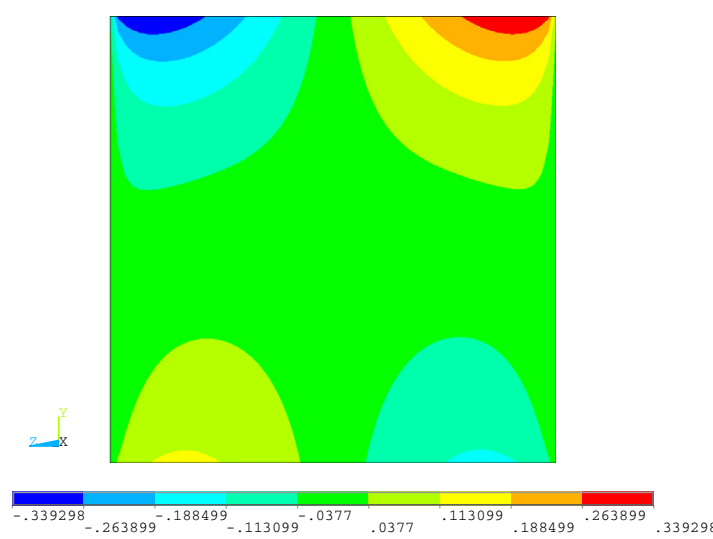

(b)

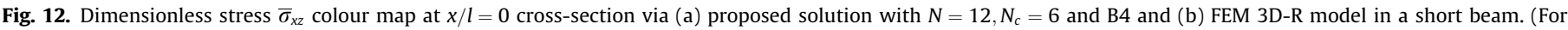
interpretation of the references to colour in this figure legend, the reader is referred to the web version of this article.)

in general terms (accuracy of the results in the case of the considered problem), whereas the Navier solution allows to assess the effect of the considered numerical solution schemes (classical FEM or FEM PGD) in the framework of the proposed beam theories. In the Navier solution, the uniform loads is approximated by its
Fourier series expansion with a number of harmonic terms that ensures the convergence of displacements and stresses up to four significant digits. Fourth- and seventh-order models yield good results for slender and short beams, respectively. Tables 3 and 4 compare the one-dimensional beam FEM and FEM PGD results 
Table 8

Computational costs of the considered solutions for a short simply supported beam.

\begin{tabular}{ll}
\hline & dofs \\
\hline FEM 3D-R $(80 \times 80 \times 80)$ & $6^{\prime} 318^{\prime} 243$ \\
FEM 3D-C $(12 \times 12 \times 12)$ & $24^{\prime} 843$ \\
FEM 1D $N=11,121$ nodes & $28^{\prime} 314$ \\
FEM 1D $N=2,121$ nodes & $2^{\prime} 178$ \\
FEM PGD 1D $N=11,121$ nodes & 597 \\
FEM PGD 1D $N=2,121$ nodes & 381 \\
\hline
\end{tabular}

for $l / a=100$ and 10 . Three (slender beam) and five (short beam) couples ensure almost coincident results between the two solution methods. Quadratic and cubic elements converge to identical results that are very close to Navier and three-dimensional results.

Tables 5-7 show the dimensionless stresses $\bar{\sigma}_{x x}, \bar{\sigma}_{y y}$ and $\bar{\sigma}_{x y}$. They are evaluated at $(l / 2, a / 2,0),(l / 2, a / 4,0)$ and $(0, a / 4,0)$, respectively. Accurate results can be obtained by the proposed models as shown by Table 5. The finite element approximation of the shear stress component $\sigma_{x y}$ via linear and quadratic elements for a slender beam is not accurate for the considered number of nodes. Accuracy can be improved by either increasing the number of nodes or by using a stress recovery technique such as the integration of the indefinite equilibrium equations, see Tornabene et al. [39]. FEM PGD results are in good agreement with the classical finite element ones. A higher number of couples than that used for displacements is required to converge to the FEM solution. Furthermore, short beams present a mechanics that is more complex than that of slender beams and they call for a higher number of couples. Table 7 shows that $\bar{\sigma}_{x x}$ and $\bar{\sigma}_{x y}$ are practically coincident between the two solution schemes for $N_{c}$ equal to six. A small difference is observed for $\bar{\sigma}_{y y}$ but $N_{c}=11$ ensures matching results for the considered number of significant digits (for instance, $\bar{\sigma}_{y y}$ is equal to 0.6383 for $N=12$ and B4 element while the corresponding classical FEM solution is 0.6385 ).

Figs. 8 and 9 qualitatively present the cross section-variation of the dimensionless displacement components $\bar{u}_{x}$ and $\bar{u}_{z}$ in the form of colour maps for a short beam. The solution obtained via a 12th-order model and six couples is compared to the FEM 3D-R model. Cross-section colour plots of dimensionless stresses $\bar{\sigma}_{x x}, \bar{\sigma}_{x y}$ and $\bar{\sigma}_{x z}$ are presented in Figs. 10-12.

The results obtained by the two different solutions are in good agreement.

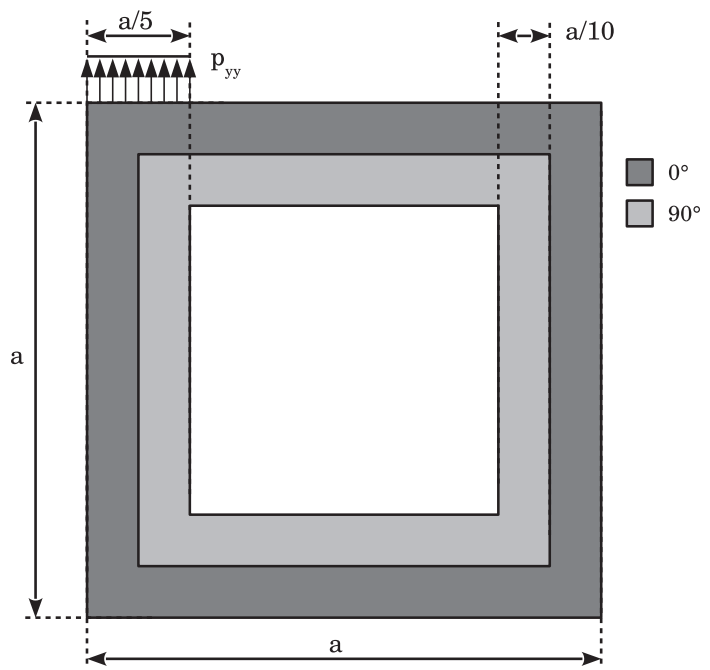

Fig. 13. Box cross-section geometry and surface bending-torsional load.
As far as the computational cost is concerned, the degrees of freedom (dof) of a three-dimensional finite element solution using serendipity quadratic Lagrangian elements are:

$d o f_{\mathrm{FEM} 3 \mathrm{D}}=3\left[N_{e x}\left(4 N_{e y}^{2}+6 N_{e y}+2\right)+3 N_{e y}^{2}+4 N_{e y}+1\right]$

where $N_{e x}$ and $N_{e y}$ are the number of elements along $x$ and $y$ and it is assumed that the number of elements along $z$ are equal to $N_{e y}$. The degrees of freedom of the Navier-type solution represent the number of unknowns per cross-section and they are $\frac{3}{2}(N+1)(N+2)$. The number of dofs of a classical one-dimensional finite element solution based upon the proposed beam models is $\frac{1}{3} N_{\mathcal{D}_{\Omega}} \cdot N_{\mathcal{D}_{x}}$ where $N_{\mathcal{D}_{\Omega}}$ and $N_{\mathcal{D}_{x}}$ have been defined in Eqs. (45) and (49), respectively. In the case of a FEM PGD one-dimensional solution, the number of dofs are $N_{\mathcal{D}_{\Omega}}+N_{\mathcal{D}_{x}}$. Table 8 presents the computational cost of the solutions used in this paper. The dofs of the FEM 3D-C solution and of the classical FEM 1D with $N=11$ are similar (although, the latter one ensures an accuracy closer to FEM 3D-R solution). This calls for solution schemes that allows for a reduction of the dofs for a given accuracy. In the case of the proposed variable separation method, the dofs of an eleventh-order one-dimensional finite element solution reduce to 597 only, whereas the accuracy remains practically the same.

\subsection{Box cross-section beam}

A $[0 / 90]$ box cross-section beam as shown in Fig. 13 is considered. The thickness of each layer is equal to $a / 10$. An off-centric pressure load $p_{y y}$ is considered in order to investigate a problem where both bending and torsion are present. Furthermore, the presence of sharp corners in the cross-section makes the prediction of mechanical response not trivial. This demanding example has been considered with the intent to assess the limits of the proposed kinematic models as well as the used numerical solution schemes. For the sake of brevity, only a short beam is considered. Table 9 presents the dimensionless displacements $\bar{u}_{x}, \bar{u}_{y}$ and $\bar{u}_{z}$. The displacements are all evaluated at $y=-a / 2$ and $z=b / 2$. The mid-span cross-section is considered for $\bar{u}_{y}$ and $\bar{u}_{z}$, whereas the axial displacement is computed at $x / l=0$. Dimensionless stresses are shown in Table 10. The axial and the shear stresses $\bar{\sigma}_{x x}, \bar{\sigma}_{x y}$ and $\bar{\sigma}_{x z}$ are computed at $(l / 2, a / 2,0),(0,0, b / 2)$ and $(0,-a / 2,0)$, respectively. One-dimensional solutions are obtained via 121

Table 9

Dimensionless displacements for a short box cross-section beam under bendingtorsion load via FEM 3D, 1D and PGD 1D solutions, 121 nodes and $N_{c}=11$.

\begin{tabular}{lllllll}
\hline & $\bar{u}_{x}$ & & $10 \times \bar{u}_{y}$ & & $\bar{u}_{z}$ & \\
\hline FEM 3D-R & 4.112 & & 4.491 & & 8.983 & \\
FEM 3D-C & 4.096 & & 4.481 & & 9.001 & \\
FEM 1D & B2 & B3, B4 & B2 & B3, B4 & B2 & B3, B4 \\
$N=14$ & 4.028 & 4.028 & 4.414 & 4.414 & 9.077 & 9.077 \\
$N=12$ & 4.023 & 4.023 & 4.395 & 4.396 & 9.104 & 9.104 \\
$N=9$ & 3.967 & 3.967 & 4.370 & 4.370 & 9.101 & 9.101 \\
$N=7$ & 4.019 & 4.019 & 4.324 & 4.324 & 9.175 & 9.175 \\
$N=4$ & 3.893 & 3.893 & 4.151 & 4.151 & 9.260 & 9.260 \\
$N=3$ & 3.907 & 3.907 & 4.000 & 4.000 & 7.732 & 7.732 \\
$N=2$ & 3.452 & 3.453 & 3.634 & 3.634 & 7.798 & 7.798 \\
FEM PGD 1D & B2 & B3, B4 & B2 & B3, B4 & B2 & B3, B4 \\
$N=14$ & 4.025 & 4.028 & 4.412 & 4.414 & 9.081 & 9.077 \\
$N=12$ & 4.023 & 4.023 & 4.395 & 4.396 & 9.105 & 9.106 \\
$N=9$ & 3.967 & 3.967 & 4.370 & 4.370 & 9.101 & 9.101 \\
$N=7$ & 4.019 & 4.019 & 4.324 & 4.324 & 9.175 & 9.175 \\
$N=4$ & 3.893 & 3.893 & 4.151 & 4.151 & 9.260 & 9.260 \\
$N=3$ & 3.907 & 3.907 & 4.000 & 4.000 & 7.733 & 7.733 \\
$N=2$ & 3.452 & 3.453 & 3.634 & 3.634 & 7.799 & 7.798
\end{tabular}

a Refined mesh: $60 \times 60 \times 120$.

b Coarse mesh: $10 \times 10 \times 10$. 
Table 10

Dimensionless stresses for a short box cross-section beam under bending-torsion load via FEM 3D, 1D and PGD 1D solutions, 121 nodes and $N_{c}=11$.

\begin{tabular}{|c|c|c|c|c|c|c|c|c|c|}
\hline & $\bar{\sigma}_{x x}$ & & & $\bar{\sigma}_{x y}$ & & & $\bar{\sigma}_{x z}$ & & \\
\hline FEM 3D-R & 2.432 & & & 5.110 & & & 2.349 & & \\
\hline FEM 3D-C ${ }^{b}$ & 2.442 & & & 5.193 & & & 2.381 & & \\
\hline FEM 1D & B2 & B3 & B4 & B2 & B3 & B4 & B2 & B3 & B4 \\
\hline$N=14$ & 24.40 & 24.41 & 24.41 & 5.221 & 5.241 & 5.233 & 2.346 & 2.349 & 2.348 \\
\hline$N=12$ & 24.45 & 24.46 & 24.46 & 5.108 & 5.132 & 5.127 & 2.421 & 2.424 & 2.422 \\
\hline$N=9$ & 24.52 & 24.53 & 24.52 & 4.837 & 4.849 & 4.839 & 2.332 & 2.335 & 2.333 \\
\hline$N=7$ & 24.64 & 24.65 & 24.64 & 4.811 & 4.829 & 4.822 & 2.404 & 2.407 & 2.405 \\
\hline$N=4$ & 25.82 & 25.82 & 25.82 & 5.130 & 5.154 & 5.143 & 2.535 & 2.538 & 2.536 \\
\hline$N=3$ & 25.84 & 25.85 & 25.84 & 4.358 & 4.395 & 4.392 & 1.581 & 1.584 & 1.583 \\
\hline$N=2$ & 24.94 & 24.95 & 24.94 & 3.055 & 3.097 & 3.098 & 1.577 & 1.580 & 1.578 \\
\hline FEM PGD 1D & B2 & B3 & B4 & B2 & B3 & B4 & B2 & B3 & B4 \\
\hline$N=14$ & 24.39 & 24.41 & 24.41 & 5.222 & 5.244 & 5.235 & 2.348 & 2.349 & 2.347 \\
\hline$N=12$ & 24.46 & 24.47 & 24.46 & 5.109 & 5.134 & 5.127 & 2.421 & 2.424 & 2.423 \\
\hline$N=9$ & 24.52 & 24.53 & 24.52 & 4.839 & 4.852 & 4.841 & 2.335 & 2.338 & 2.336 \\
\hline$N=7$ & 24.64 & 24.65 & 24.64 & 4.813 & 4.830 & 4.822 & 2.407 & 2.410 & 2.409 \\
\hline$N=4$ & 25.81 & 25.82 & 25.82 & 5.130 & 5.154 & 5.143 & 2.535 & 2.538 & 2.536 \\
\hline$N=3$ & 25.84 & 25.85 & 25.84 & 4.358 & 4.394 & 4.391 & 1.581 & 1.584 & 1.583 \\
\hline$N=2$ & 24.94 & 24.95 & 24.94 & 3.055 & 3.097 & 3.098 & 1.577 & 1.580 & 1.578 \\
\hline
\end{tabular}

a Refined mesh: $60 \times 60 \times 120$.

Coarse mesh: $10 \times 10 \times 10$

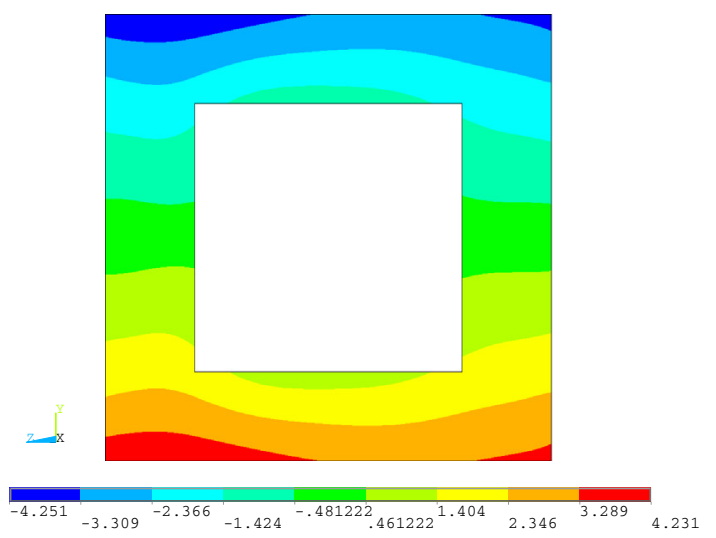

(a)

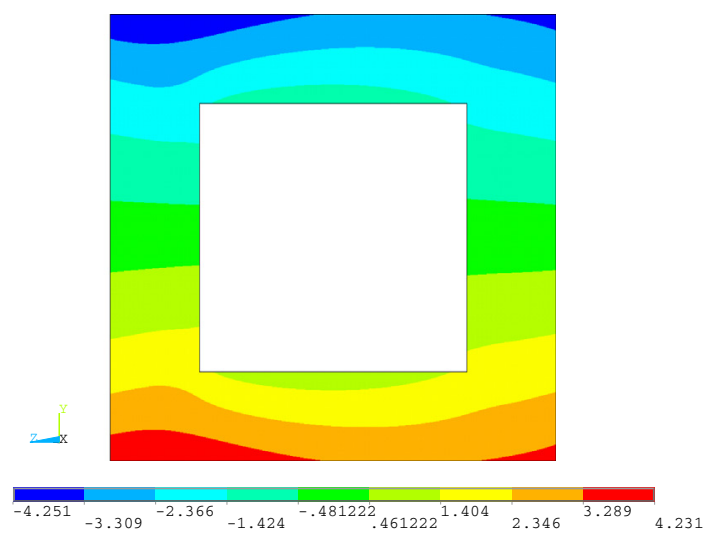

(b)

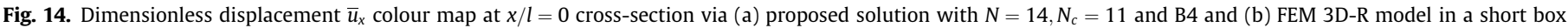
beam. (For interpretation of the references to colour in this figure legend, the reader is referred to the web version of this article.)

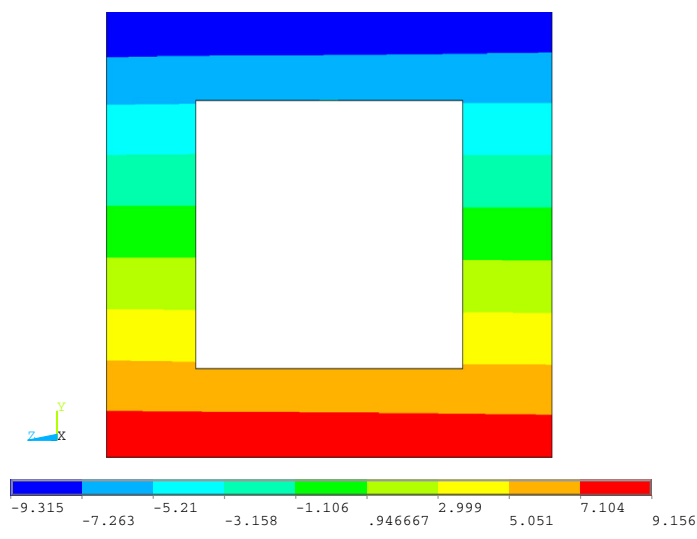

(a)

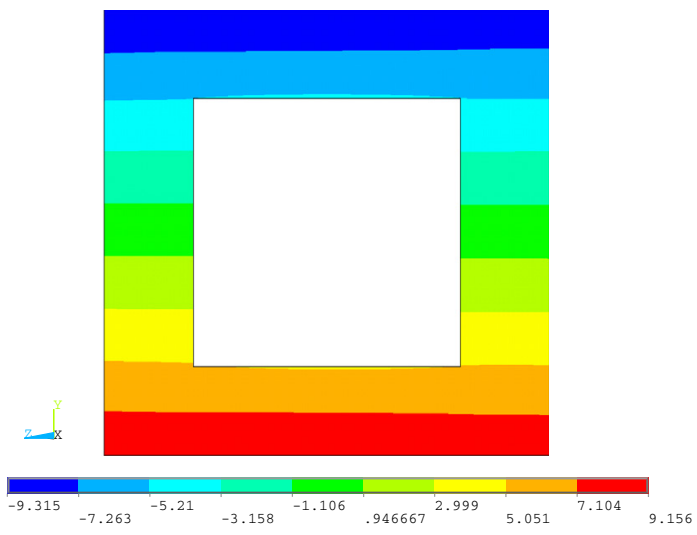

(b)

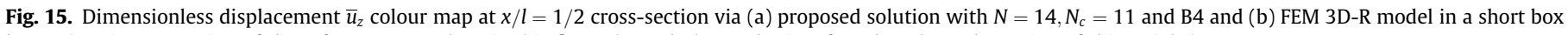
beam. (For interpretation of the references to colour in this figure legend, the reader is referred to the web version of this article.) 


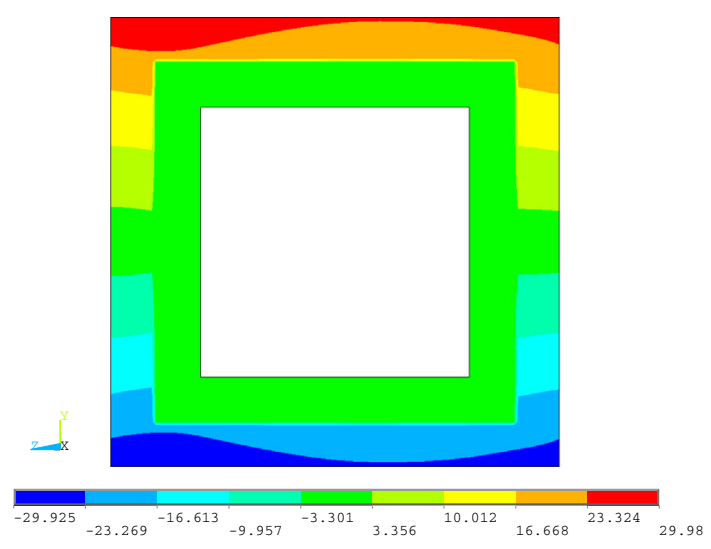

(a)

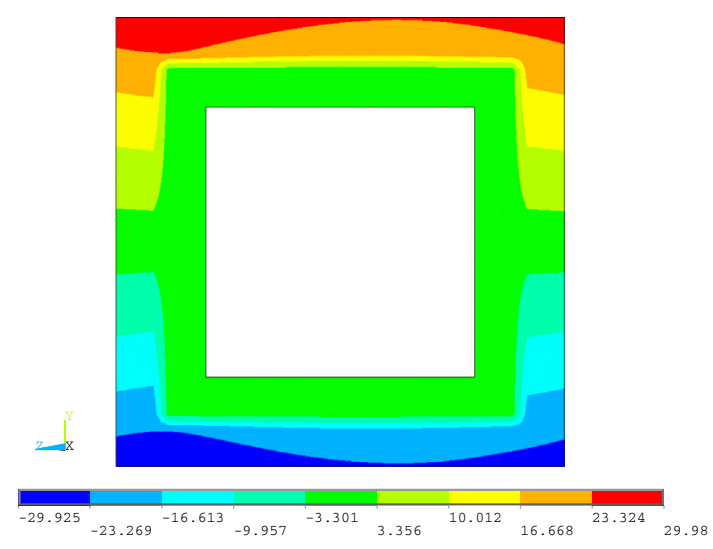

(b)

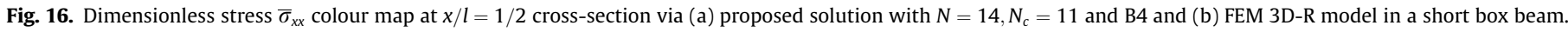
(For interpretation of the references to colour in this figure legend, the reader is referred to the web version of this article.)

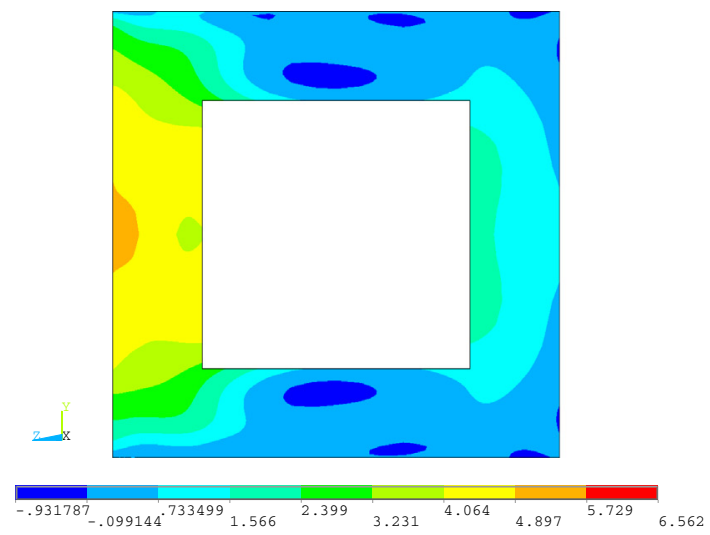

(a)

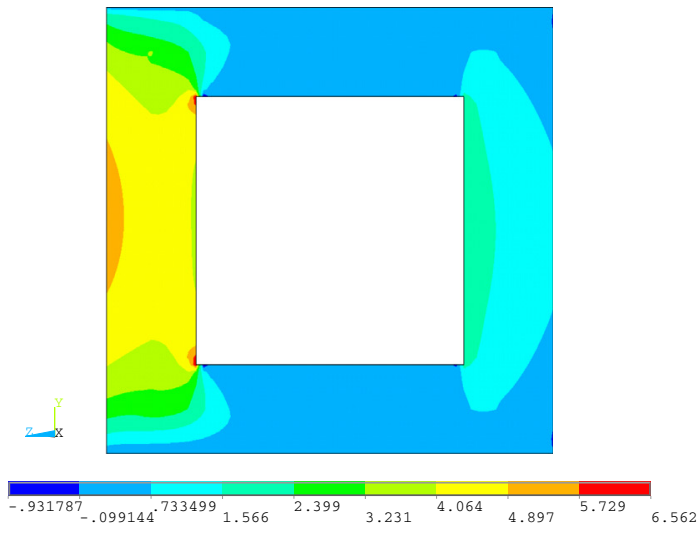

(b)

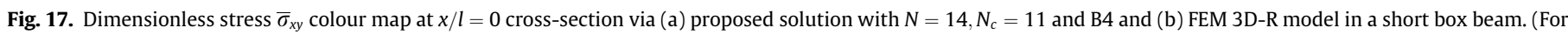
interpretation of the references to colour in this figure legend, the reader is referred to the web version of this article.)

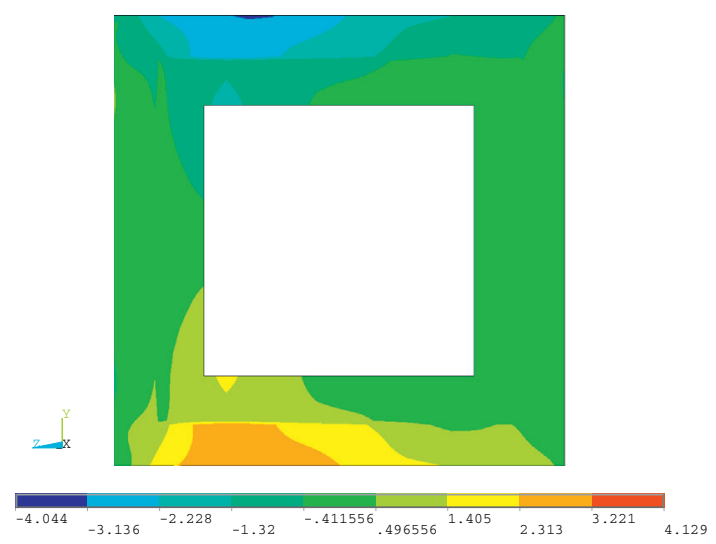

(a)

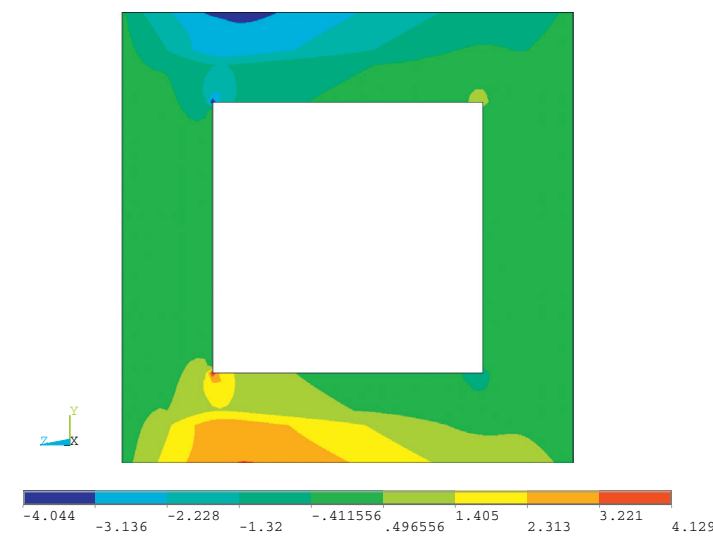

(b)

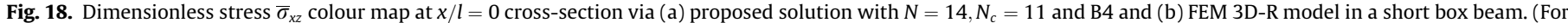
interpretation of the references to colour in this figure legend, the reader is referred to the web version of this article.)

nodes and 11 couples are used for the domain decomposition. Results show that 11 couples ensures a good agreement classical FEM and FEM PGD solution, being the results very close. As far as the proposed kinematic models are concerned, a fair accuracy can be observed. Figs. 14-18 present displacements and stresses variation over the cross-section. The reference FEM 3D-R solution is compared to a FEM 1D PGD model with $N=14$ and $N_{c}=11$. Results fairly agree for the displacements and the axial stress component. In the case of the shear stresses, stress concentrations are present in the internal sharp corners of the cross-section making 
the solution difficult to be accurately predicted. It should be observed that this limitation is due to the kinematic model. Accurate results can be obtained by changing the manner displacements are approximated over the cross-section. Future work will use a layer-wise modelling approach, see Carrera et al. [19]. In this manner, the displacement field can be locally refined resulting in improved results for a given number of degrees of freedom.

\section{Conclusion}

In this paper, a hierarchical family of higher-order one-dimensional beam finite elements has been developed in the framework of a Proper Generalised Decomposition, a variables separation method that allows reducing the computational costs inherent to higher-order models. The displacement field has been approximated over the cross-section via a Unified Formulation in order to derive several kinematic models able to account for non-classical effects such as shear deformation and in- and out-of-plane warping. Furthermore, finite elements with a general number of nodes per element along the beam axis can be obtained via this Unified Formulation. Linear, quadratic and cubic Lagrangian element have been derived. The global problem has been divided into two coupled ones over the cross-section and along the beam axis via the adopted variables separation method. The dimensions of the resulting problems are considerably smaller than that of an equivalent classical finite element solution. This approach is particular appealing in the case of higher-order solutions with a refined mesh along the beam axis since a high amount of computer volatile memory is required and stuck overflow can occur. The developed elements have been used to investigate the static response of three-dimensional laminated beam structures. Simply supported beams with square prismatic or channelled box cross-sections have been investigated. Analyses accounted for both slender and short beams subjected to bending as well as bending-torsional loads. Results have been validated through comparison with analytical Navier-type solutions and three-dimensional finite element solutions obtained via the commercial code ANSYS. The presented results showed that quasi three-dimensional solutions for both displacement and stress components can be obtained in a very computational effective manner. Some limitations in the kinematic field have been observed in the case of a short beam with a laminated box cross-section under a bending-torsion load due to the internal sharp corners. Nevertheless, the used generalised decomposition is able to converge to the classical corresponding finite element solution provided a suitable number of couples is considered. Future work will focus on the improvement of the displacement field by a layer-wise approach in order to refine the solution locally.

\section{References}

[1] Giunta G, Metla N, Koutsawa Y, Belouettar S. Free vibration and stability analysis of three-dimensional sandwich beams via hierarchical models. Compos Part B: Eng 2013;47:326-38.

[2] Kapania RK, Raciti S. Recent advances in analysis of laminated beams and plates, Part I: Shear effects and buckling. AIAA J 1989;27(7):923-34.

[3] Kapania RK, Raciti S. Recent advances in analysis of laminated beams and plates, part ii: Vibrations and wave propagation. AIAA J 1989;27(7):935-46.

[4] Ghugal YM, Shimpi RP. A review of refined shear deformation theories for isotropic and anisotropic laminated beams. I Reinf Plast Compos 2001;20(3):255-71.

[5] Chakravarty UK. On the modeling of composite beam cross-sections. Compos Part B: Eng 2011:42(4):982-91.

[6] Maiti DK, Sinha PK. Bending and free vibration analysis of shear deformable laminated composite beams by finite element method. Compos Struct 1994:29:421-31.

[7] Shimpi RP, Ghugal YM. A layer-wise trigonometric shear deformation theory for two-layered cross-ply laminated beams. J Reinf Plast Compos 1999; $18: 1516-42$
[8] Shimpi RP, Ghugal YM. A new layer-wise trigonometric shear deformation theory for two-layered cross-ply beams. Compos Sci Technol 2001;61:1271-83.

[9] Matsunaga H. Interlaminar stress analysis of laminated composite beams according to global higher-order deformation theories. Compos Struct 2002;55:105-14.

[10] Hodges DH, Atilgan AR, Cesnik CES, Fulton MV. On a simplified strain energy function for geometrically nonlinear behaviour of anisotropic beams. Compos Eng 1992;2(5-7):513-26.

[11] Yu W, Hodges DH, Volovoi V, Cesnik CES. On Timoshenko-like modeling of initially curved and twisted composites beams. Int J Solids Struct 2002;39(19):5101-21.

[12] Vo TP, Lee J. Geometrically nonlinear theory of thin-walled composite box beams using shear-deformable beam theory. Int J Mech Sci 2010;52(1):65-74.

[13] Lin X, Zhang YX. A novel one-dimensional two-node shear-flexible layered composite beam element. Finite Elem Anal Des 2011;47(7):676-82.

[14] Aguiar RM, Moleiro F, Mota Soares CM. Assessment of mixed and displacement-based models for static analysis of composite beams of different cross-sections. Compos Struct 2012;2(94):601-16.

[15] Vo TP, Thai H-T. Static behavior of composite beams using various refined shear deformation theories. Compos Struct 2012;94(8):2513-22.

[16] Erkmen RE. Multiple-point constraint applications for the finite element analysis of shear deformable composite beams - variational multiscale approach to enforce full composite action. Comput Struct 2015;149:17-30.

[17] Carrera E. Theories and finite elements for multilayered plates and shells: a unified compact formulation with numerical assessment and benchmarking. Arch Comput Meth Eng 2003;10(3):215-96.

[18] Carrera E, Giunta G. Exact, hierarchical solutions for localised loadings in isotropic, laminated and sandwich shells. J Press Vess Technol 2009;131(4):041202.

[19] Carrera E, Giunta G, Petrolo M. Beam structures: classical and advanced theories. John Wiley and Sons; 2011.

[20] Giunta G, Biscani F, Belouettar S, Carrera E. Analysis of thin-walled beams via a one-dimensional unified formulation through a Navier-type solution. Int J Appl Mech 2011;3(3):407-34.

[21] Catapano A, Giunta G, Belouettar S, Carrera E. Static analysis of laminated beams via a unified formulation. Compos Struct 2011;94(1):75-83.

[22] Castellanos JA, Larrazabal G. A Cholesky out-of-core factorization. Math Comput Model 2013;57(9-10):2207-22.

[23] Ammar A, Mokdad B, Chinesta F, Keunings R. A new family of solvers for some classes of multidimensional partial differential equations encountered in kinetic theory modeling of complex fluids. J Nonnewton Fluid Mech 2006;3(139):153-76.

[24] Nouy A. A priori model reduction through proper generalized decomposition for solving time-dependent partial differential equations. Comput Meth Appl Mech Eng 2010;199(23-24):1603-26.

[25] Chinesta F, Ammar A, Leygue A, Keunings R. An overview of the proper generalized decomposition with applications in computational rheology. J Nonnewton Fluid Mech 2011;166(11):578-92.

[26] Savoia M, Reddy J. A variational approach to three-dimensional elasticity solutions of laminated composite plates. J Appl Mech 1992;59(2S):166-75.

[27] Bognet B, Bordeu F, Chinesta F, Leygue A, Poitou A. Advanced simulation of models defined in plate geometries: 3d solutions with $2 \mathrm{~d}$ computational complexity. Comput Meth Appl Mech Eng 2012;201-205:1-12.

[28] Vidal P, Gallimard L, Polit O. Composite beam finite element based on the proper generalized decomposition. Comput Struct 2012;102-103:76-86.

[29] Vidal P, Gallimard L, Polit O. Assessment of a composite beam finite element based on the proper generalized decomposition. Compos Struct 2012;94(5):1900-10.

[30] Vidal P, Gallimard L, Polit O. Proper generalized decomposition and layer-wise approach for the modeling of composite plate structures. Int J Solids Struct 2013;50(14-15):2239-50.

[31] Vidal P, Gallimard L, Polit O. Explicit solutions for the modeling of laminated composite plates with arbitrary stacking sequences. Compos Part B: Eng 2014:60:697-706.

[32] Vidal P, Gallimard L, Polit O. Shell finite element based on the proper generalized decomposition for the modeling of cylindrical composite structures. Comput Struct 2014:132:1-11.

[33] Gallimard L, Vidal P, Polit O. Coupling finite element and reliability analysis through proper generalized decomposition model reduction. Int J Numer Meth Eng 2013;95(13):1079-93.

[34] Reddy JN. Mechanics of laminated composite plates and shells. Theory and analysis. CRC Press; 2004.

[35] Horn RA. The Hadamard product. In: Proceedings of symposia in applied mathematics. vol. 40; 1990. p. 87-169.

[36] Bathe KJ. Finite element procedure. Prentice Hall; 1996.

[37] Carrera E, Giunta G, Nali P, Petrolo M. Refined beam elements with arbitrary cross-section geometries. Comput Struct 2010;88(5-6):283-93.

[38] ANSYS v12.0 theory manual. ANSYS Inc., Southpointe, PA; 2009.

[39] Tornabene F, Liverani A, Caligiana G. Static analysis of laminated composite curved shells and panels of revolution with a posteriori shear and normal stress recovery using generalized differential quadrature method. Int J Mech Sci 2012;61(1):71-87. 